\title{
Confusion in cosmology and gravitation
}

\section{${ }^{1,2,3}$ Christian Corda, ${ }^{4}$ R. Katebi and ${ }^{5}$ N. O. Schmidt}

June 24, 2015

\author{
${ }^{1}$ Dipartimento di Fisica, Scuola Superiore di Studi Universitari e Ricerca \\ "Santa Rita", via Trasaghis 18/E, 00188 Roma, Italy
}

${ }^{2}$ Austro-Ukrainian Institute for Science and Technology, Institut für Theoretishe Physik, Technische Universität, Wiedner Hauptstrasse 8-10/136, A-1040, Wien, Austria

${ }^{3}$ International Institute for Applicable Mathematics \& Information Sciences (IIAMIS), B.M. Birla Science Centre, Adarsh Nagar, Hyderabad - 500 463, India

\footnotetext{
${ }^{4}$ Department of Physics and Astronomy, Ohio University, Athens, Ohio 45701, USA

${ }^{5}$ Department of Mathematics, Boise State University, 1910 University Drive, Boise, ID 83725, USA
}

Correspondence should be addressed to C. Corda; cordac.galilei@gmail.com

\begin{abstract}
In a series of papers, Santilli and collaborators released various strong statements against the general theory of relativity (GTR) and the standard $\Lambda$ CDM model of cosmology. In this paper we show that such claims are due to fundamental misunderstandings of very basic concepts of gravitation and cosmology. In other words, we show that Santilli and collaborators demonstrated nothing. In particular, they demonstrated neither that the GTR is wrong, nor that the Universe is not expanding. We also show that the so-called iso-gravitation theory (IGT) of Santilli is in macroscopic
\end{abstract}


contrast with geodesic motion and, in turn, with the Equivalence Principle (EP) and must therefore be ultimately rejected. Finally, we show that, although the so called iso-redshift could represent an interesting alternative (similar to the tired light theory historically proposed by Zwicky) to the Universe expansion from a qualitative point of view, it must be rejected

from a quantitative point of view because the effect of iso-redshift is $10^{-6}$ smaller than the effect requested to achieve the cosmological redshift.

Paper dedicated to the 80th birthday of Ruggero M. Santilli, hoping that this will permit Santilli to understand how GTR and $\Lambda$ CDM cosmology really work and, in turn, will permit him to withdraw his very wrong claims in gravitation and cosmology.

Keywords: gravitation; cosmology; Equivalence Principle; expansion of the Universe.

\section{Introduction}

In a series of papers [1]-[6], Santilli claims to have found various flaws in Einstein's GTR [30] and that such flaws can be corrected through his so-called IGT [3]-[5]. In this paper we clarify that such supposed flaws are strong misconceptions by Santilli on the basic concepts of the GTR instead. In particular, Santilli does not know and/or does not understand the EP, on which, not only the GTR, but all the metric theories of gravity are founded [7]. We also show that the IGT is not viable, for at least two fundamental reasons. The first is theoretical, the latter experimental. From the theoretical point of view, Santilli [5] claims indeed that his theory is founded on the Freud Identity of Differential Geometry [8]. We show that Santilli's interpretation of the Freud Identity is wrong from both the mathematical and physical points of views. In fact, on one hand Santilli mathematically confuses true tensors with pseudo-tensors. This issue was previously clarified in [9], but here we reexamine it from a slightly different point of view, using the definitions of tensors and pseudo-tensors in the classical book [10]. This reexamination is necessary because Santilli and collaborators insist in a wrong mathematical interpretation of the Freud Identity in recent works $[11,12]$, generating further confusion. On the other hand, Santilli physically does not take into account the EP.

From the experimental point of view, the absence of space-time curvature in the IGT implies a macroscopic contrast with geodesic motion and, in turn, with the EP $[7,13]$ which is today tested with a precision of order $10^{-14}[14,15]$. We also stress that, starting from the historical experiments by Loránd Eötvös in 1890 [16], which tested the equivalence between inertial and gravitational mass with a precision of order $10^{-8}$, the EP has been tested with always increasing precision; today the EP stands as a fundamental principle of nature [17]. This means that the IGT is in very strong contrast with tons of data collected in more than a century and therefore must be ultimately rejected.

In another series of papers and announcements [18, 22], Santilli and collaborators claim to have found various flaws in the standard $\Lambda \mathrm{CDM}$ model of 
cosmology and that the expansion of the Universe can be dismissed through the so-called iso-redshift. In this paper we also clarify that such supposed flaws are strong misconceptions by Santilli and collaborators on the basic concepts of the standard $\Lambda \mathrm{CDM}$ model of cosmology. In particular, the claim that "Hubble's law establishes that the cosmological redshift is the same for all galaxies having the same distance from Earth in all directions in space. Consequently, the conjectures on the expansion of the universe, the acceleration of the expansion and the big bang necessarily imply a return to the Middle Ages with Earth at the center of the universe", or similar claims such as those in [18, 22], are well known to be completely wrong at the popularizing level of physics folklore and at the high school level. At such levels the similarity between the Universe's expansion and the surface of an expanding balloon - which has no center - is explained [23]. From a technical point of view, the Universe is seen as a spacelike hyper-surface having no center. Also, the so-called "conjectures" are not conjectures. Instead, they are applications of metric theories of gravity (which are the only viable applications, based on the extremely well-tested precision of the EP [7]), starting from the GTR) to the cosmological observations and to the Cosmological Principle which states that "that the distribution of matter in the Universe is homogeneous and isotropic when viewed on a large enough scale" [10, 24], i.e. exactly the opposite of Santilli's wrong claim that the Earth is the center of the Universe. Finally, we also show that, although the so-called iso-redshift $[18,22]$ could, in principle, represent an alternative (similar to the tired light theory historically proposed by Zwicky [25]) to the Universe expansion from a qualitative point of view, it must be rejected from a quantitative point of view because the effect of iso-redshift is $10^{-6}$ smaller than the effect requested to achieve the cosmological redshift.

\section{Confusion in gravitation}

For the sake of clearness, we recall that Santilli calls "Einstein gravitation" the vacuum Einstein field equations while he calls "Einstein general relativity" the Einstein field equation in presence of sources [1]-[6], [11, 59]. After this clarifiyng, we can proceed with our analysis.

Some of Santilli's wrong claims are historical. For example, in [11], which is basically a review of [1]-[6], Santilli claims that being in the year 2015, we are "in connection with the centennial of the first geometric conception of gravitation". This is wrong. It is indeed well known that the GTR, of which this year is the centennial, was not the first geometric theory of gravitation. Historically, the relativistic scalar theory of gravitation introduced in 1912-13 by the Finnish physicist Gunnar Nordström in [26]-[28], has been the first geometric theory of gravity. In fact, it was derived three years before Einstein's GTR. This is well known in various papers throughout the literature, see for example the recent paper [29]. This is not the sole wrong historical mistake by Santilli and collaborators, as we will see in the following. In [11, 59] Santilli also claims that "Einstein general relativity is a scientic religion". This is completely wrong 
and unacceptable. It is indeed well known that, although Einstein's GTR [30] achieved great success (see for example the opinion of Landau who says that GTR is, together with quantum field theory, the best scientific theory of all [10]) and withstood many experimental tests [7, 17], it also displayed many shortcomings and flaws which today make theoreticians question whether it is the definitive theory of gravity [29], [31]-[33]. Differently from other field theories like the electromagnetic theory, the GTR has not yet been quantized. This issue avoids treating gravitation like other quantum theories, precluding, in turn, the unification of gravitation with the other interactions. On the other hand, one defines Extended Theories of Gravity (ETG) as a group of semi-classical theories having the Lagrangian modified with respect to the standard Einstein-Hilbert gravitational Lagrangian. Such modifications include the addition of high-order terms in the curvature invariants (terms like $R^{2}, R^{\alpha \beta} R_{\alpha \beta}, R^{\alpha \beta \gamma \delta} R_{\alpha \beta \gamma \delta}, R \square R$, $R \square^{k} R$ ) or of terms with scalar fields being non-minimally coupled to the geometry (terms like $\phi^{2} R$ ) [29], [31]-[33]. In fact, these kind of terms are usually considered in various approaches that attempt to perform the unification between gravitation and the other interactions. Another important issue is that, from the cosmological point of view, such extensions of GTR can generate inflationary approachs that result in very important attempts to solve various problems of the standard Universe model, starting from the historical work of Starobinsky [34]. We stress that, differently from Santilli [1]-[6] [11], we are not claiming that the GTR is wrong. It is well known that, even in the general framework of ETG, the GTR continues to serve as the most important part of the structure [29], [31]-[33]. The ETG approach only attempts to understand if (and how) weak modifications to the GTR structure can help to solve some theoretical and observational problems [29], [31]-[33]. Historically, the same Einstein claimed that the GTR structure could not be definitive [35]. It is indeed well known that, during his famous research on the unified field theory in the latest years of his life, Einstein attempted to realize a theory that he called the generalized theory of gravitation, but he claimed that mathematical difficulties prevented him from obtaining the final equations [35].

Considering the general context of cosmological observations, one also finds other considerations and approaches that suggest extensions to the GTR [29], [31]-[33]. In fact, today the Universe appears appears to be undergoing a period of accelerated expansion. The cosmological dynamics seem to be dominated by the so-called Dark Energy, which gives a large negative pressure [36]-[40]. This new ingredient in the standard picture is considered as a source of the right side of the Friedmann-Lemaitre-Robertson-Walker (FLRW) field equations. In the standard Universe model the global dynamics are driven by this un-clustered non-zero vacuum energy together with the clustered Dark Matter [36]-[40]. The global framework is called the "concordance model" $(\Lambda \mathrm{CDM})$ and gives, together with the CMBR, LSS and SNeIa data, the most general accepted tapestry of the Universe as it is observed today. On the other hand, $\Lambda \mathrm{CDM}$ cosmology shows various shortcomings as the well known "coincidence" and "cosmological constant" problems [40]. The alternative approach of ETG changes the left side of the field equations instead, attempting to achieve the observed cosmic 
dynamics through the extension of the GTR [29], [31]-[33]. In the ETG context we do not need to search candidates for Dark Energy and Dark Matter, that, currently, have not yet been found. Only the "observed" ingredients, being curvature and baryon matter, have to be taken into account. Thus, one can think that gravity could be different at different scales [41] and there is room for alternative theories. As a result, various Dark Energy and Dark Matter models can be achieved considering $f(R)$ theories of gravity, where $R$ is the Ricci curvature scalar, and/or scalar-tensor gravity (STG) [29], [31]-[33], where STG is a generalization of the historical Brans-Dicke theory [42]. It has been shown in [31] that if advanced projects on the detection of gravitational waves (GWs) will improve their sensitivity - allowing to perform a GWs astronomy through accurate angular and frequency dependent response functions of interferometers for GWs arising from various theories of gravity, i.e. GTR and ETG - then this will be the ultimate test for the GTR.

We stress that we have been forced to insert this digression on the ETG to falsify Santilli's claim that "Einstein general relativity is a scientic religion" [11].

Another claim by Santilli is that there is a "lack of clear compatibility of general relativity with special relativity" [1]-[6], [11]. This is another elementary mistake. There is no lack of clear compatibility of the GTR with the special relativity theory (SRT) if one possesses a thorough understanding of the EP, which is any of the various connected concepts involving, on one hand, the equivalence of inertial and gravitational mass, and on the other hand, the observation by Einstein that the gravitational "force" that an observer experiences locally when standing in the gravitational field generated by a massive body (for example the Earth) is equivalent to the pseudo-force experienced in an accelerated (non-inertial) reference frame [24]. One of the various interpretations of the EP implies that a globally curved space-time is locally flat [24]. In other words, gravitational effects are always locally negligible and, in a local Lorentz frame, where the space-time can be considered flat in an excellent approximation, the SRT works very well [24].

Santilli defines the "First historical insufficiency of general relativity" as "Ignoring the refraction of star light passing through the Sun chromosphere, with consequential lack of evidence that space is curved" [11, 59]. That Santilli calls "historical" this and the following supposed insufficiences of GTR is a mystery and completely new to us. In our knowledge Santilli is indeed the sole person who claims that GTR has these supposed insufficiences. In any case, here are the various misconceptions.

1. It is wrong that the GTR claims that "the 0.87 arc-seconds deviation is caused by Newton gravitation" and "the remaining 0.87 arc-seconds deviation have been known for a century to be due to the curvature of space" $[11,59]$. Instead, the GTR demonstrates that the whole 1.75 arc-second "bending" of star light passing near the Sun is due to the space-time curvature, not to the curvature of space [24]. In the GTR the global space-time is curved, not only the spatial surfaces [24]. In fact, the GTR is not based 
on spatial curvature but on space-time curvature [24]. There are indeed solutions - for example the cosmological ones with $k=0$ - where, although the spatial section is Euclidean at constant time, the global space-time structure is curved, i.e. it is not Lorentzian, but only conformally flat [24].

2. Claiming that " 0.87 arc-seconds deviation is caused by Newton gravitation" and "The remaining 0.87 arc-seconds deviation is due to to the refraction of star light when passing through the Sun chromosphere" [11, 59] is very wrong for various reasons. First of all Newton gravitation admits instantaneous propagation, and this is in contrast with the SRT, as this theory requests interactions to have finite velocities of propagation. Second, today the bending of the light is carefully tested for many stars which are very far from the Sun chromosphere. The entire sky is indeed slightly distorted due to the gravitational deflection of light caused by the Sun (except the anti-Sun direction ). This effect has been observed by the European Space Agency astrometric satellite Hipparcos [43]. It measured the positions of about $10^{5}$ stars [43]. The results agree with the prevision of the GTR at the level of 0.3 percent [43]. Clearly, as the Newtonian value is exactly half of the Einsteinian one, a precision of the level of 0.3 percent rules out in an ultimate way the possibility to consider the gravitational bending of the light in a purely Newtonian context. Third, the Sun chromosphere was very different in the past and it will be very different in the future. Thus, if the author should be correct the consequence should be that, as the contribution of the Sun chromosphere is exactly the same as that of the contribution of the Newtonian theory at the present time, we are currently living in a very special period in the history of the solar system, because that contribution was very different in the past and will be very different in the future. Clearly, this issue cannot be taken seriously into account.

3. It is well known that also the Newtonian theory of gravity can be written in the language of curved space-time [24]. In fact, the EP is not unique to the GTR description of the concepts of gravity [24]. What is unique to Einstein's vision is the combination of the EP with the local Lorentz geometry [24]. Let us return to the Newtonian "universal time" [24]. For the trajectories of test particles, the Newtonian theory of gravity gives [24]

$$
\frac{d^{2} x^{j}}{d t^{2}}=\frac{\partial V}{\partial x^{j}},
$$

where $V$ is the Newtonian potential. The most famous interpretation of the Newtonian gravitational theory is that eq. (1) describes the "curved paths" $x^{j}(t)$ along which test particles move in the flat Euclidean space (not space-time) [24]. On the other hand, there exists an alternative description, which is due to Cartan [24], which interprets the trajectories of eq. (1) as geodesics $\left[t(\lambda), x^{j}(\lambda)\right]$ in curved space-time [24]. Details of 
this important issue, which still remark that the claims by Santilli against curvature are wrong, can be found in [24]. Here we limit ourselves to recall that the source equation for the Newtonian potential

$$
\nabla^{2} V \equiv \sum_{j} V_{, j j}=-4 \pi \rho
$$

can be rewritten in the geometric form [24]

$$
R_{00}=4 \pi \rho,
$$

where $R_{\alpha \beta}$ is the Ricci tensor.

On the other hand, and contrary to the Santilli's claims in [1]-[6], [11, 59], the bending from the light by the Sun is not considered to be the most important experimental proof that space-time is curved. Instead, the most important experimental proof is the EP. Before clarifying this point, we need to stress another important issue. Santilli claims in [11] that the inertial mass is bigger than the gravitational mass and explicity wrote down this point in eq. (7) of [11]. This is in strong contrast with tons and tons of experimental and observational data. Starting from the famous, historical, experiments by Eötvös in 1908, who found that the difference is less than 1 part in $10^{8}$ [16], we have the experiments by Roll, Krotkov and Dicke, who found that the difference is less than 1 part in $10^{11}$ [44], and the experiments by Braginsky and Panov [45], who found that difference is less than 1 part in $10^{12}$, etc. To date, the most precise experimental results are those obtained by Baessler et al. [14, 15], who found that, if a difference should exist, it must be less than 1 part in $10^{14}$. In our knowledge, the equivalence between inertial and gravitational mass is one of the most precise instances of experimental evidence in the whole history of science, and it is today considered to be a fundamental principle of nature [17]. Thus, even admitting that eq. (7) of [11] is correct, the difference between the inertial mass and the gravitational mass must be less than 1 part in $10^{14}$ in order to not contradict the experimental results (we stress that Santilli and collaborators always stressed the Galileian statement that a scientist must never assume positions in contrast with experimental results $[46,47]$ in accordance to the scientific method). On the other hand, such a difference is completely negligible in the framework of classical gravitational theories.

After having clarified this fundamental issue, now we explain why today the EP is considered to be the most important experimental proof that space-time is curved. The key point is that the EP implies that test masses must follow geodesic lines. This point is very intuitive, but has been also rigorously demonstrated by Weinberg [13]. Before writing the derivation of this fundamental issue we stress its important consequence: in the absence of space-time curvature geodesic motion is given by straight lines! But instead, of course, all astrophysical observations show that the gravitational motion is not given by straight lines. Hence, the only possibility is that space-time is curved. In other words, Santilli's assumption of the absence of space-time curvature should therefore indicate a macroscopic violation of the equivalence between the inertial mass and 
the gravitational mass, which, instead, is tested with the enormous precision of 1 part in $10^{14}[14,15]$. Clearly, considering also the experiments $[16,44,45]$ etc., it is obvious that Santilli's claim of the absence of space-time curvature is in very strong contrast with tons of data collected in more than a century. Now, let us show that the EP implies that test masses must follow geodesic lines. This will be also an excellent example of how dynamical equations and causal structures arise from observations. We stress that in the following derivation we closely follow [13]. Let us start supposing that no particles are accelerating in the neighborhood of a point-event with respect to a freely falling coordinate system $\left(X^{\mu}\right)$ [13]. Putting $T=X^{0}$ one writes down the following equation that is locally applicable in free fall [13]

$$
\frac{d^{2} X^{\mu}}{d T^{2}}=0
$$

Using the chain rule one gets [13]

$$
\frac{d X^{\mu}}{d T}=\frac{d x^{\nu}}{d T} \frac{\partial X^{\mu}}{\partial x^{\nu}}
$$

Differentiating eq. (5) with respect to $T$ one gets [13]

$$
\frac{d^{2} X^{\mu}}{d T^{2}}=\frac{d^{2} x^{\nu}}{d T^{2}} \frac{\partial X^{\mu}}{\partial x^{\nu}}+\frac{d x^{\nu}}{d T} \frac{d x^{\alpha}}{d T} \frac{\partial^{2} X^{\mu}}{\partial x^{\nu} \partial x^{\alpha}} .
$$

Combining eqs. (4) and (6) one immediately gets [13]

$$
\frac{d^{2} x^{\nu}}{d T^{2}} \frac{\partial X^{\mu}}{\partial x^{\nu}}=-\frac{d x^{\nu}}{d T} \frac{d x^{\alpha}}{d T} \frac{\partial^{2} X^{\mu}}{\partial x^{\nu} \partial x^{\alpha}}
$$

Multiplying both sides of eq. (7) by $\frac{\partial x^{\lambda}}{\partial X^{\mu}}$ one gets [13]

$$
\frac{d^{2} x^{\lambda}}{d T^{2}}=-\frac{d x^{\nu}}{d T} \frac{d x^{\alpha}}{d T}\left[\frac{\partial^{2} X^{\mu}}{\partial x^{\nu} \partial x^{\alpha}} \frac{\partial x^{\lambda}}{\partial X^{\mu}}\right]
$$

Setting $t=x^{0}$ and using again the chain rule, $T$ can be eliminated in favor of the coordinate time $t[13]$

$$
\frac{d^{2} x^{\lambda}}{d t^{2}}=-\frac{d x^{\nu}}{d t} \frac{d x^{\alpha}}{d t}\left[\frac{\partial^{2} X^{\mu}}{\partial x^{\nu} \partial x^{\alpha}} \frac{\partial x^{\lambda}}{\partial X^{\mu}}\right]+\frac{d x^{\nu}}{d t} \frac{d x^{\alpha}}{d t} \frac{d x^{\lambda}}{d t}\left[\frac{\partial^{2} X^{\mu}}{\partial x^{\nu} \partial x^{\alpha}} \frac{\partial x^{0}}{\partial X^{\mu}}\right] .
$$

Recalling that the bracketed terms involving the relationship between local coordinates $X$ and general coordinates $x$ are functions of the general coordinates, eq. (9) gives immediately the geodesic equation of motion using the coordinate time $t$ as parameter) [13]

$$
\frac{d^{2} x^{\lambda}}{d t^{2}}=-\Gamma_{\nu \alpha}^{\lambda} \frac{d x^{\nu}}{d t} \frac{d x^{\alpha}}{d t}+\Gamma_{\nu \alpha}^{0} \frac{d x^{\nu}}{d t} \frac{d x^{\alpha}}{d t} \frac{d x^{\lambda}}{d t}
$$

which is equivalent to the standard geodesic equation written in terms of the scalar parameter $s[13]$

$$
\frac{d^{2} x^{\lambda}}{d s^{2}}=-\Gamma_{\nu \alpha}^{\lambda} \frac{d x^{\nu}}{d s} \frac{d x^{\alpha}}{d s}
$$


Clearly, based on the extreme precision on which the EP is today tested and verified, the demonstration that we have reviewed here - i.e. that geodesic motions arise from the EP - ultimately rules out Santilli's IGT, which is instead founded on the absence of curvature. Notice that, based on our criticisms [58], Santilli attempted to take into due account the EP in [59] claiming that "This raises the question as to whether Einstein's Equivalence Principle also holds for exterior isogravitation with a source. Einstein supporters quickly voice their opinion that this is not the case for the intent of invalidating isogravitation" and that "In particular, it is easy to see that Einstein's Equivalence Principle is maintained in its integrity in multiple ways. First of all, the projection of isogravitation on the conventional Riemannian space over a conventional field coincides with Einstein gravitation with consequential trivial validity of Einstein's Equivalence Principle. Additionally, the Equivalence principle independently holds also on the Minkowski-Santilli isospace over isofield by very conception of isotopies". Again, Santilli misunderstands the key point. The problem is not the potential contrast between the EP and the "exterior isogravitation with a source". As we have shown above, instead the real problem is that the absence of curvature is in macroscopic contrast with the EP. In addition, some of Santilli's claims contradict each other. In fact on one hand Santilli claims that the vacuum Einstein field equations are wrong, see [1]-[6], [11, 59] and the below discussions. On the other hand, in order to attempt preserving the EP, he is forced to reduce the IGT to the vacuum Einstein field equations [59].

Other misconceptions by Santilli result from the very wrong claims that "I $r$ respective of the above, the conjecture of curvature of space has been unable to represent without ambiguities truly basic gravitational events, such as the free fall of masses that has to be necessarily along a "straight" radial line, the weight of bodies in a gravitational field, and other basic events that are clearly represented by Newtonian gravitation" [11]. Of course, these are very elementary mistakes. First of all, we stress that a fundamental constraint used not only by Einstein in the derivation of the field equations [10, 24, 30], but also by Schwarzschild [48] in the derivation of his famous and fundamental solution to the Einstein field equations in vacuum was that, in the weak field approximation, i.e. at large distances, the general relativistic gravitational field must reduce to the Newtonian gravitational field. Clearly, the weak field approximation works very well near the Earth's surface. Thus, neglecting the higher-order terms in the general relativistic gravitational field and considering the Newtonian approximation permits to recover both of the free fall of masses, the weight of bodies in a gravitational field, and all of the other basic events of Newtonian gravitation. Second, again Santilli does not understand how the EP works. As the motion obeys the geodesic of eq. (11), locally a geodesic becomes a "straight" radial line and the free fall of masses is completely found also in the full GTR. In other words, for short distances where the gravitational field can be considered constant, the geodesics of a curved space-time are extremely well approximated by "straight" radial lines of a flat space-time in the same way that a curve line is locally well approximated by a straight line. This is another consequence of Einstein Equivalence Principle: in an inertial frame of reference bodies (and light) 
obey Newton's first law, moving at constant velocity in straight lines. Analogously, in a curved spacetime the world line of an inertial particle or pulse of light is as straight as possible (in space and time) [88]. On the other hand, the issue that the weight of bodies in a gravitational field can be achieved also by the full GTR has been well explained in [49].

Another wrong claim by Santilli is that "Despite one century of studies, the "actual" orbits of planets in our Solar system have not been represented in an accurate, unique and time invariant way via Einstein gravitation, while they are exactly and unambiguously represented by Newton's gravitation and Kepler's laws. In fact, calculations based on the Riemannian geometry of the actual orbits of planets, besides not being unique due to the non-linearity of the theory, are different than physical orbits, and are not the same over time". To falsify this misconception let us write down the Schwarzschild line element in geometrized units as [24]

$$
d s^{2}=\left(1-\frac{2 M_{S}}{r}\right) d t^{2}-r^{2}\left(\sin ^{2} \theta d \varphi^{2}+d \theta^{2}\right)-\frac{d r^{2}}{1-\frac{2 M_{S}}{r}}
$$

where $M_{S}$ is the solar mass. Setting $\theta=\frac{\pi}{2}$ in order to consider test bodies moving in the "equatorial plane" one computes to order $\frac{M_{S}}{r}$ the shape $r(\varphi)$ of the nearly Keplerian, nearly elliptical geodesic orbit as [24]

$$
r=\frac{\left(1-e^{2}\right) a}{1+e \cos \left[\left(1-\frac{\delta \varphi_{0}}{2 \pi}\right) \varphi\right]},
$$

where $e$ and $a$ are constants of integration and [24]

$$
\delta \varphi_{0}=\frac{6 \pi M_{S}}{\left(1-e^{2}\right) a} .
$$

Contrary to Santilli's claims and based on Birkhoff's theorem [24], it is well known that the line element of eq. (12) is unique despite the non-linearity of the theory, see also [50] for further details. Eq. (13) is more precise than the corresponding Newtonian counterpart which is

$$
r=\frac{\left(1-e^{2}\right) a}{1+e \cos \varphi}
$$

corresponding to $\delta \varphi_{0}=0$. In fact, eq. (13) takes into due account the presence of the precession (14). On the other hand, eq. (15), which is the Newtonian limit of eq. (13), is a perfect Keplerian ellipse having the semi-major axis $a$ and eccentricity $e$ [24].

Santilli also claims that the "second historical insufficiency of general relativity is ignoring the electromagnetic origin of the mass, with consequential invalidation of Einstein's reduction of gravitation to pure curvature without sources" [11]. Here Santilli generates massive confusion and attempts to propagate it. He indeed claims "to have identified the electromagnetic origin of the mass via the 
full use of quantum electrodynamics, including advanced and retarded treatments and showed that such an origin requires the necessary presence in the r.h.s. of the field equations of source tensor of first order in magnitude, irrespective of whether the body is charged or neutral" [11]. In other words, Santilli claims that the vacuum Einstein field equations are

$$
G_{i k}=R_{i k}-\frac{R}{2} g_{i k}=0, \quad \text { with } \quad i, j,=1,2,3,4,
$$

where $G_{i k}, R_{i k}, R$ and $g_{i k}$ are the Einstein tensor, the Ricci tensor, the Ricci scalar and the metric tensor, respectively (see [10, 13, 24] for details), and are in contrast with his equations [11]

$$
G_{i j}=k T_{i j, e l m},
$$

where Santilli claims that $k$ is a unit-dependent constant and $T_{i j, e l m}$ should be the "source tensor of first order in magnitude" arising from quantum electrodynamics [11]. The terms "first order in magnitude" should be "referred to the condition of entirely representing the gravitational mass of the body considered" [11]. In particular Santilli claims that "the mass of the electron is of entirely electromagnetic origin" and, as a consequence, the vacuum Einstein field equations of eq. (16) should be insufficient to represent the gravitational field of the electron in favor of his eq. (17) [11].

First of all, we stress that the GTR is a classical theory, which by definition does not take into account quantum effects. On the other hand, we show that, even considering the tensors found by Santilli in a classical approach, Santilli is wrong. In fact, within a classical framework we stress that vacuum is vacuum, i.e. we cannot force a source tensor to be always present in vacuum. To clarify this issue, we proceed as follows. Let us consider the electron in the framework of classical theories in terms of a sphere having the classical Compton radius $r_{e} \cong 2.8 * 10^{-15}$ meters. We use this approach because the Compton radius is much greater than the Planck length, i.e. $r_{e} \gg l_{p} \cong 1.6 * 10^{-35}$ meters, and we know that the GTR breaks down at the Planck scale [24]. Thus, assuming spherical symmetry and the correctness of Santilli's stress-tensor $T_{i j, e l m}$, we have the following framework for the field equations of the electron's gravitational field:

$$
\begin{gathered}
\text { unknown for } 0 \leq r \leq l_{p} \text { (we need a theory of quantum gravity) } \\
G_{i k}=k T_{i j, e l m} \quad \text { for } l_{p} \leq r \leq r_{e} \\
G_{i k}=0 \quad \text { for } r \geq r_{e} .
\end{gathered}
$$

In other words, assuming that Santilli's field equations of eq. (17) are correct, they are not in contrast with Einstein's field equations of eq. (16). One must merely use eq. (17) in the electron's interior and eq. (16) for the external geometry. We stress that the vacuum Einstein field equations sometimes generate 
some confusion because one can ask: how is it possible that eq. (16) works? In other words, how is it possible that a gravitational field can exist without a source? This key point is clarified in a very enlightening way by t' Hooft in [51], verbatim "Einstein's equations are non-linear, and this is why gravitational fields can be the source of an additional amount of gravity, so that a gravitational field can support itself." In other words, it is the non-linear part of eq. (16) which acts as a self-source of the gravitational field.

Santilli attempts to endorse his strong misunderstanding of this above discussed issue by claiming that the Freud identity of differential geometry [8] should establish "the need on purely mathematical grounds of a source tensor of first order in magnitude in the r.h.s of the field equations" according to his eq. (17) [11]. This is another misconception that we clarify immediately, but before we stress that such a misconception has been clarified from a purely mathematical point of view in [9]. As previously emphasized above, we now reanalyze this issue from a slightly different point of view with respect to [9], by using the definitions of tensors and pseudo-tensors in the classical book [10]. As Santilli and collaborators insist in wrong mathematical and physical interpretations of the Freud Identity in the recent works [11, 12, 59] (which generate further confusion), this reanalyzing is necessary. In addition we also discuss the physical counter part of the mathematical examination, which is missed in [9], which again concerns the EP.

Santilli $[1,6,11]$ claims that eq. (16) violates the Freud identity of differential geometry $[8]$ which is $[8,9]$

$$
\begin{aligned}
& 2 \mathfrak{U}_{\kappa}^{\iota}=\delta_{\kappa}^{\iota}\{\left\{\sqrt{-g}\left[R+g^{\mu \nu}\left(\Gamma_{\mu \sigma}^{\rho} \Gamma_{\rho \nu}^{\sigma}-\Gamma_{\mu \nu}^{\rho} \Gamma_{\rho \sigma}^{\sigma}\right)\right]\right\}-2-\sqrt{-g} R_{\kappa}^{\iota} \\
&+\left[\Gamma_{\mu \nu}^{\iota} \partial_{\chi}\left(\sqrt{-g} g^{\mu \nu}\right)-\Gamma_{\mu \nu}^{\nu} \partial_{\chi}\left(\sqrt{-g} g^{\mu \iota}\right)\right],
\end{aligned}
$$

with $[8,9]$

$$
\mathfrak{U}_{\mu}^{\nu} \equiv \frac{\partial}{\partial x_{\rho}} \mathfrak{U}_{\mu}^{\nu \rho},
$$

being $[8,9]$

$$
\mathfrak{U}_{\mu}^{\nu \rho} \equiv \sqrt{-g} S_{\mu}^{\nu \rho}
$$

the Freud superpotentials, with $[8,9]$

$$
S_{\mu}^{\lambda \rho} \equiv \frac{1}{2} \operatorname{det}\left[\begin{array}{ccc}
\delta_{\mu}^{\lambda} & \delta_{\mu}^{\sigma} & \delta_{\mu}^{\iota} \\
g^{\lambda \kappa} & g^{\sigma \kappa} & g^{\iota \kappa} \\
\Gamma_{\kappa \iota}^{\lambda} & \Gamma_{\kappa \iota}^{\sigma} & \Gamma_{\iota \kappa}^{\iota}
\end{array}\right],
$$

see $[8,9]$ for details.

Rearranging eq. (19), Santilli writes down the Freud identity as (eq. (3.10) in $[6])$

$$
\begin{aligned}
& R_{\beta}^{\alpha}-\frac{R}{2} \delta_{\beta}^{\alpha}-\frac{1}{2} \Theta \delta_{\beta}^{\alpha} \\
= & U_{\beta}^{\alpha}+\frac{\partial V_{\beta}^{\alpha \rho}}{\partial x^{\rho}}=k T_{\beta}^{\alpha},
\end{aligned}
$$


where

$$
\Theta \equiv g^{\alpha \beta} g^{\gamma \delta}\left(\Gamma_{\rho \alpha \beta} \Gamma_{\gamma \beta}^{\rho}-\Gamma_{\rho \alpha \beta} \Gamma_{\gamma \delta}^{\rho}\right)
$$

and $U_{\beta}^{\alpha}$ and $V_{\beta}^{\alpha \rho}$ are defined in eqs. (3.11b) and (3.11c) of [6]. On the other hand, in [9] Notte-Cuello and Rodrigues claim that in his rearranging Santilli missed a term in eq. (23) and that eq. (23) must be corrected as [9]

$$
\begin{gathered}
R_{\beta}^{\alpha}-\frac{R}{2} \delta_{\beta}^{\alpha}-\frac{1}{2} \Theta \delta_{\beta}^{\alpha} \\
=U_{\beta}^{\alpha}+\frac{\partial V_{\beta}^{\alpha \rho}}{\partial x^{\rho}}+S_{\beta}^{\alpha \rho} \Gamma_{\rho \sigma}^{\sigma}=k T_{\beta}^{\alpha} .
\end{gathered}
$$

Setting $T_{\beta}^{\alpha}=0$ in eq. (23), Santilli claims that that equation is in contrast with the vacuum Einstein field equations of eq. (16) $[6,11]$ and that "unfortunately, the Freud identity was not aligned with Einstein's doctrines and, as such, the identity was virtually ignored in the entire literature on gravitation of the 20-th century" [6]. We show that, independently on which is the correct equation between eqs. (23) and (25) (we think the latter is correct), both of Santilli's claims are wrong. In fact, using the definition of eq. (24) and the definition of the Einstein tensor, setting $T_{\beta}^{\alpha}=0$ eqs. (23) and (25) obviously imply

$$
G_{\beta}^{\alpha}=R_{\beta}^{\alpha}-\frac{R}{2} \delta_{\beta}^{\alpha}=\frac{1}{2} \delta_{\beta}^{\alpha}\left[g^{\alpha \beta} g^{\gamma \delta}\left(\Gamma_{\rho \alpha \beta} \Gamma_{\gamma \beta}^{\rho}-\Gamma_{\rho \alpha \beta} \Gamma_{\gamma \delta}^{\rho}\right)\right]
$$

But we recall that the Christoffell symbols (and, in turn, the quantity $\Theta$ defined in eq. (24)) in eq. (26) are not true tensors [10]. They are pseudo-tensors instead [10]. In fact, differently than true tensors - which when being equal to zero in a coordinate system must be equal to zero in every other one [10] - the Christoffell symbols become null in a local Lorentz coordinate system, but, in a curvilinear space, it is impossible to make all the Christoffell symbols null over all of space [10]. Thus, in a local Lorentz coordinate system one gets $\Theta=0$. This obviously implies that the Einstein tensor is null in eq. (26). On the other hand, the Einstein tensor is a true tensor. Thus, when being equal to zero in a coordinate system it must be equal to zero over all of space. This implies that eq. (26) becomes

$$
G_{\beta}^{\alpha}=0 \quad \text { over all of space, }
$$

which are the vacuum Einstein field equations. Thus, in complete agreement with [9] and in contrast with Santilli's wrong claims, we have shown that there is no contrast between the Freud identity of differential geometry and the vacuum Einstein field equations from the mathematical point of view. In other words, the Freud identity of differential geometry is a further strong proof that the vacuum Einstein field equations are correct. Now, let us clarify which is the physical counterpart of the above mathematical examination. The key point is the following. In the framework of metric theories of gravity, the components of the metric tensor are considered to be the potentials, while the Christoffell symbols are considered to be the components of the gravitational field [10]. Thus, we still evoke the EP which states that one can always choose a coordinate 
system, i.e. local Lorentz coordinate system, where the gravitational field is null. This again sets all the Christoffell symbols equal to zero and eq. (27) follows immediately from eq. (26). On the other hand, the issue that the Einstein tensor is null over all of space does not imply that the space is flat. To obtain that condition we need the stronger constraint that the Riemann tensor must be null over all of space [10].

We note that Santilli also claims that the Freud identity should be consistent with his eq. (17) $[1,6,11]$. This is again wrong. In fact, such a consistency should imply

$$
k T_{i j, e l m}=\frac{1}{2} \delta_{\beta}^{\alpha}\left[g^{\alpha \beta} g^{\gamma \delta}\left(\Gamma_{\rho \alpha \beta} \Gamma_{\gamma \beta}^{\rho}-\Gamma_{\rho \alpha \beta} \Gamma_{\gamma \delta}^{\rho}\right)\right] .
$$

But, again, we stress that the quantity on the r.h.s. of eq. (28) is not a true tensor, but it is a pseudo-tensor instead. As a consequence, in a local Lorentz coordinate system one gets

$$
T_{i j, e l m}=0,
$$

and, as the stress-energy tensor is a true tensor, eq. (29) must be equal to zero over all of space. Thus, inserting the constraint of eq. (29) in eq. (17), one immediately finds the vacuum Einstein field equations of eq. (27). We note that, also in this case, based on our criticisms [58] Santilli has been forced to attempt to put a patch on his claims. In fact, in [59] he claims that "As a final note, the reader may have noted the lack of use of the mathematical terms "tensors" or "pseudotensors" and the use instead of the physical term "source." This is due to the fact that the clear physical content of the forgotten Freud identity is often dismissed on the ground of purely mathematical differences in nomenclatures and personal mathematical interpretations". These claims are completely unscientific. It is well known - and we rigorouly demonstrated this issue above that there is an enormous difference between tensors and pseudo-tensors from both the mathematical and physical points of view. In particular, a "source" cannot be a pseudo-tensor if we want it to equal the Einstein tensor, which is a true tensor. We stress again that the physical counterpart of the mathematical difference between tensors and pseudo-tensors is given by the EP - a fact that Santilli evidently does not understand. In other words, behind the difference between tensors and pseudo-tensors there is the most important foundation, not only of the GTR, but of the whole framework of metric theories of gravity. If one does not understand this fundamental point, he cannot understand how GTR and the broader framework of metric theories of gravity work. In fact, the correct mathematical and physical interpretations of the Freud identity of differential geometry that we discussed above is the second strong reason supporting the case that Santilli's IGT must be ultimately rejected. Santilli indeed claims in $[2,6]$ to have "assumed the Freud identity as the geometric foundation of the $I G T$ ". But we have shown that Santilli's interpretation of the Freud identity is wrong from both the mathematical and physical points of view. Thus, a theory founded on such strong misconceptions cannot be correct.

Santilli claims that "the third historical insufficiency of GTR is abandoning the majestic Lorentz and Poincare "invariance" of special relativity in favor of 
the "covariance" of general relativity with consequential lack of prediction of the same numerical values under the same conditions at different times" [11]. This claim is very obscure although Santilli attempted to clarify it in [6]. In that work, Santilli claims that "Riemannian theories of gravitation in general, and Einstein's gravitation in particular, can at best describe physical reality at a fixed value of time, without a consistent dynamic evolution". This is, of course, completely wrong. The GTR is well known to be a causal theory having a perfect dynamic evolution. In fact, all the known vacuum solutions, which are obtained assuming rigorous boundary constraints, are static (Schwarzschild [52] and Reissner-Nordström solutions [53, 54]) or stationary (Kerr, Kerr Newman [24] and gravitational waves solutions [55]). Of course, claiming that static or stationary solutions miss "a consistent dynamic evolution" [6] is unscientific. On the other hand, it is also known that solutions in the presence of matter - such as the cosmological solutions that we will discuss in the next section and the stellar models [56, 57] - are rigorously deterministic. In order to endorse his wrong claims, Santilli attempts to release an example claiming that "suppose that a Riemannian theory predicts a numerical value at the initial time $t=$ 0 , such as the $43^{\prime \prime}$ for the precession of the perihelion of Mercury. One can prove that the same prediction at a later time $t=0$, is numerically different precisely in view of the 'covariance', rather than invariance as intended in special relativity, thus preventing a serious application of the theory to physical reality". In order to dismiss this wrong claim it is sufficient to note that the result of eq. (14) concerning the precession of the perihelion of Mercury is completely time independent.

We also recall that general covariance arises from the idea that coordinates are not present, a priori, in the physical world. They are only artifices used in describing the physical world, and therefore they do not play a concrete role in the formulation of the physical laws governing the nature of the Universe. This idea is exactly the opposite of what is claimed by Santilli. In addition, not only are the GTR and the other metric theories of gravity formulated in terms of general covariance, but also the classical (non-quantum) theory of electrodynamics which is well known to be a rigorous deterministc theory and does not suffer the problems supposed by Santilli. General covariance extends the SRT's Lorentz invariance - which can be applied only to inertial frames - to the more general case which can be applied to all frames. In fact, upon evoking the EP, which implies that space-time is locally flat, we hence locally retrieve Lorentz invariance as a particular case of general covariance.

Another claim by Santilli is that [59] "Another serious insufficiency is that the description by general relativity of the "exterior gravitational problem" in vacuum is incompatible with the "interior gravitational problems" that dominated the scientific scene in gravitation until the advent of Einstein's theory. This is a serious incompatibility because its resolution prohibits the use of the Riemannian geometry due to the need of a geometry not only without curvature, but also with a metric having a dependence on coordinates $x$, as well as density $m \mu$, temperature $\tau$, frequency $\omega$, etc. $g=g(x, \mu, \tau, \omega, \ldots)$ ". Concerning the supposed need of the absence of curvature, we have previously shown that 
Santilli is wrong. On the other hand, it is well known that the existence of solutions in the presence of matter (interior gravitational problems) - such as, for example, the cosmological solutions that we will discuss in the next section and the solutions for the stellar models $[56,57]$ - work very well in the presence of curvature. In addition, even admitting that Santilli is correct in claiming that we need a metric having a dependence on coordinates, density, temperature, frequency, etc., Santilli does not clarify why such a metric should be in contrast with the Riemannian geometry. On the other hand, starting from the historical works of Schwarzschild $[52,60]$, it has been shown various times that it is possible to match the internal and external geometries in a satisfactory way for various cases of physical importance, starting from static stars and arriving to the gravitational collapse - see $[24,50]$ for details.

Let us discuss a further wrong claim by Santilli, which concerns a misunderstanding of the Parametrized Post Newtonian (PPN) approximation. For this, Santilli indeed claims that "for any claim of "experimental verification" (of the vacuum Einstein field equations) we can assume a different PPN approximation with different expansions and show dramatic divergences of Einstein general relativity from physical realities" [59] and that "the vacuum Einstein field equations admit a variety of inequivalent expansions, depending on the selected parameter, the selected expansion and the selected truncation. It is then easy to show that the selection of an expansion of the same vacuum Einstein field equations but different from the PPN approximation leads to dramatic departures from experimental values". Santilli does not understand the real meaning of the Post Newtonian Parameters (PNPs). Differently from his wrong claims, the vacuum Einstein field equations (16) admits a unique PPN approximation in terms of well-fixed PNPs. The running of parameters is used to discriminate among the GTR and the other metric theories of gravity through gravitational experiments [7, 17, 24]. In fact, Will [7] clarified that "The comparison of metric theories of gravity with each other and with experiment becomes particularly simple when one takes the slow-motion, weak-field limit. This approximation, known as the post-Newtonian limit, is sufficiently accurate to encompass most solar-system tests that can be performed in the foreseeable future". If one analyzes Table 2 of [7] concerning the PNPs, one can immediately see that all the PNPs are fixed in the GTR. In particular, only 2 - which are known as the Eddington-Robertson-Schiff Parameters [7] - which are among the 10 PNPs are different from zero, i.e. $\gamma=\beta=1$ [7]. The simplest example of using the PPN approximation is to again consider the gravitational deflection of light. The formula can be indeed written in terms of the sole $\gamma$ as [7]

$$
\delta \theta=\frac{1}{2}(1+\gamma) \frac{4 M_{s}}{d} \frac{1+\cos \Phi}{2},
$$

where $M_{S}$ is the solar mass, and $\Phi$ is the angle between the Earth-Sun line and the incoming direction of the photon [7]. Setting the standard values $d \simeq d_{s}$ and $\Phi \simeq 0$ one gets $[7]$

$$
\delta \theta \simeq \frac{1}{2}(1+\gamma) 1.7505 \text { arc }- \text { second } .
$$


In this way, one can obtain very precise values of $\gamma$ through very precise measurements of the gravitational deflection of light using the formula

$$
\gamma=\frac{2 \delta \theta}{1.7505 \text { arc }- \text { second }}-1 .
$$

Thus, if one gets $\gamma=1$ the GTR is confirmed. Instead, if one finds deviations from $\gamma=1$ there is room for alternative metric gravitational theories. This is the case of the above discussed ETG. On the other hand, this cannot be the case of Santilli's IGT in [1]-[6], [11, 59] as we have above shown that this theory is founded on misconceptions on the Freud identity of differential geometry [8] and on the absence of curvature, which generates macroscopic violations of the EP.

Let us highlight another important issue. By using the power of the EP, the framework of the GTR permits one to ultimately explain the Mössbauer rotor experiment [61], dismissing the claims by Santilli [62] and Kholmetskii et al. [63], who claimed that only the so-called Santilli's iso-mathematics can explain the Mössbauer rotor effect. Let us summarize this issue. A historical experiment by Kündig [64] on the transverse Doppler shift in a rotating system measured with the Mössbauer effect (Mössbauer rotor experiment) has been recently first reanalyzed [65] and then replied [66] by an experimental research group. The results of reanalyzing the experiment have shown that a correct reprocessing of Kündig's experimental data gives an interesting deviation of a relative redshift between emission and absorption resonant lines from the standard prediction based on the relativistic dilatation of time [65]. That prediction gives a redshift $\frac{\nabla E}{E} \simeq-\frac{1}{2} \frac{v^{2}}{c^{2}}$ where $v$ is the tangential velocity of the absorber of resonant radiation, $c$ is the velocity of light in vacuum and the result is given to the accuracy of first-order in $\frac{v^{2}}{c^{2}}[61,65]$. Data re-processing gave $\frac{\nabla E}{E} \simeq-k \frac{v^{2}}{c^{2}}$ with $k=0.596 \pm 0.006$ [65]. Subsequent new experimental results by the reply of Kündig experiment have shown a redshift with $k=0.68 \pm 0.03$ instead [66]. By using the power of the EP, which states the equivalence between the gravitational "force" and the pseudo-force experienced by an observer in a non-inertial frame of reference (included a rotating frame of reference) in [61] the theoretical framework of the Mössbauer rotor experiments has been reanalyzed directly in the rotating frame of reference by using a general relativistic treatment. It has been shown that previous analyses missed an important effect of clock synchronization and that the correct general relativistic prevision in the rotating frame gives $k \simeq \frac{2}{3}$ in perfect agreement with the new experimental results [61]. Such an effect of clock synchronization has been missed in various papers in the literature with some subsequent claim of invalidity of relativity theory and/or some attempts to explain the experimental results through the so-called Santilli's iso-mathematics [62, 63]. The general relativistic interpretation in [61] shows, instead, that the new experimental results of the Mössbauer rotor experiment are a new, strong and independent, proof of the GTR. For the sake of completeness we recall that the Mössbauer effect (discovered by R. Mössbauer in 1958 [67]) consists in resonant and recoil-free emission and absorption of gamma rays, without loss of energy, by atomic nuclei bound in a solid. Its past and 
current results are very important for basic research in physics and chemistry. In [61] - [67] the authors focused on the so-called Mössbauer rotor experiment. In this particular experiment, the Mössbauer effect works through an absorber orbiting around a source of resonant radiation (or vice versa) [61] - [67]. The aim is to verify the relativistic time dilation time for a moving resonant absorber (the source) inducing a relative energy shift between emission and absorption lines [61] - [67].

Finally, we finish this section by clarifying some wrong claims by Santilli against the existence of gravitational waves (GWs). This will also be a sort of introduction for the next section, where we will clarify and correct the wrong claims by Santilli and collaborators in Cosmology. Concerning GWs, Santilli claimed that [11] "Various colleagues have brought to my attention the recent withdrawal from publication of claims of "experimental verification" of gravitational waves, as discussed in articles at The Economist, Scientific American, and in other conduits. To my knowledge, this is the first glimpse of scientific sanity in one hundred years of Einstein gravitation, because all preceding claims of "experimental verifications" were instantly published by biased editors without even a lilliputian image of the galactic severity used in the review of opposing claims, both editorial reviews generally being without a serious or otherwise credible scientific content. Somewhat encouraged by a possible return of gravitation to scientific sanity, I decided to indicate that the impossibility to date of detecting gravitational waves is much deeper than what stated, since gravitational waves are prohibited by the historical insufficiencies of Einstein gravitation that have remained ignored in the mainstream literature for one century" and that "the theoretical prediction of gravitational waves will remain in a kind of "suspended animation" until the electromagnetic origin of the gravitational mass is dismissed in refereed publications". Let us start to clarify that, contrary to Santilli's claims, the BICEP 2 results [68] have not been withdrawn from publication. What really happened is that there are alternative interpretations on those results. For example, interstellar dust physics can generate contamination for $\mathrm{CMB}$ polarization experiments with the consequence that, verbatim from [69], "there are no "clean" windows where primordial CMB B-mode polarization could be measured without subtraction of dust emission". We stress that, although the BICEP 2 results are still a controversial issue, it concerns the relic GWs, which are the weakest GW signal that today researchers are attempting to detect, see for example [29, 70]. On the other hand, it is well known that there is a strong indirect proof of the existence of GWs through the compact binary system composed by two neutron stars PSR1913+16 [71]. This is the famous Hulse and Taylor pulsar, which not only permitted the authors of [71] to win the 1993 Nobel Prize in physics, but it has also been, for physicists working in the GWs research field, the ultimate thrust that has paved-the-way to achieve the extremely sophisticated technology needed for investigating on the GWs physics [72]. On the other hand, we recall that in the above discussions we have ultimately shown that the so-called "historical insufficiencies of Einstein gravitation" (by Santilli) are instead strong misconceptions and that we have ultimately dismissed that "the electromagnetic origin of the gravitational mass" 
should be in contrast with the vacuum Einstein field equations and with the presence of the curvature of space-time, contrary to Santilli's wrong claims.

\section{Confusion in cosmology}

The claim by Santilli that "As it is well known to historians, Albert Einstein, Edwin Hubble, Fritz Zwicky, Enrico Fermi, Louis de Broglie, and other famous scientists died without accepting the conjecture of the expansion of the Universe because it implies a necessary return to the Middle Ages with Earth at the center of the Universe" [22] is completely new to us. It is indeed very difficult to think that all of these very famous scientists similarly made this elementary mistake that is well known to be completely wrong at the popularizing level of physics folklore and at the high school level. In fact, at such levels the similarity between the Universe's expansion and the surface of an expanding balloon which has no center - is explained [23]. On the other hand, it is unacceptable that Santilli releases such a strong incorrect statement without inserting precise citations, as he did in [22]. In any case, we are sure that the above claim by Santilli is wrong, at least for Albert Einstein. In fact, although it is well known that Einstein had various doubts on the expansion of the Universe, his relationship with cosmology was very different. In 1917 he proposed a temporally infinite but spatially finite model as his preferred cosmological model [73]. In that work, he introduced the famous cosmological constant, $\Lambda$, as an addition to the GTR to "hold back gravity", i.e. to force the equations to predict a static universe. In fact, Einstein originally did not realize that his GTR predicts the Universe's expansion and he inserted the cosmological constant to avoid a collapsing Universe. It is curious to observe that such a mistake by Einstein is today remade by Santilli and collaborators who indeed claim that "according to Einstein general relativity, as a result of which the explanation of the acceleration of the expansion of the universe (rather than the expected contraction due to gravitational attraction requires yet additional theoretical conjectures)" [19] an that "As a matter of fact, according to Einstein general relativity, in the event the universe had been composes in a numerically predominant way of any form of energy, the universe should contract, and definitely should not expand" [22]. This is of course wrong, as we will show in the following discussion. In any case, Einstein was the referee of the historical paper by Friedmann, who pioneered the theory that the Universe was expanding and governed by the GTR field equations [74]. It is fascinating to see that historically Friedmann found that the Universe was expanding in 1924 [74], i.e. before Hubble's observations [75]. It was G. Lemaitre who found that Hubble's observations were in full agreement with the Friedmann model of an expanding Universe based on the GTR, and again this happened before Hubble's observations [76]. In fact, Lemaître derived Hubble's law and made the first estimation of the Hubble's constant in a completely theoretical framework in 1927, i.e. two years before Hubble's paper [76]. The theory by Friedmann and Lemaitre was further refined by H. P. Robertson [77] and, independently, by A. G. Walker [78]. Thus, the cosmological solution 
of the GTR field equations that describes a homogeneous and isotropic Universe is today called the Friedmann-Lemaittre-Robertson-Walker (FLRW) solution, or the Big-Bang Theory, and is still considered the basis of modern cosmology. Einstein was aware of all the developments that we described above and, according to G. Gamow [79], such developments led him to reverse his opinion on the Universe's static behavior and to claim that his cosmological model in [73] - and especially the introduction of the cosmological constant - his "biggest blunder". Clearly, Einstein cannot have made the elementary mistake of Santilli and collaborators [19] - [22] that "the expansion of the Universe implies a necessary return to the Middle Ages with Earth at the center of the Universe" as he studied the work of Friedmann, Lemaître, Robertson and Walker which was founded on the GTR field equations (in geometrized units) [24]

$$
G_{i k}=8 \pi T_{i k}
$$

and he was well aware that such works were founded on the so-called Cosmological Principle, which states that "the distribution of matter in the Universe is homogeneous and isotropic when viewed on a large enough scale" [24], i.e. exactly the opposite of the wrong claims by Santilli and collaborators in [19] - [22]. At a fixed instant of the cosmic time, the Universe is indeed seen as a space-like hyper-surface having no centre [24]. In similar way, we are very perplexed that the other great scientists cited by Santilli and collaborators, i.e. "Edwin Hubble, Fritz Zwicky, Enrico Fermi, Louis de Broglie, and other famous scientists" [19] - [22] really claimed that "the expansion of the Universe implies a necessary return to the Middle Ages with Earth at the center of the Universe". We also stress that the term "Big-Bang" was coined by F. Hoyle on 28 March 1949, on BBC Radio's Third Programme broadcast [80]. Hoyle intended to be pejorative, as it is well known that Hoyle was one of the proponents of the "steady state" model of the Universe (steady state theory) [81], together with T. Gold, H. Bondi [82] and Hoyle's student J. Narlikar [83]. We emphasize that the steady state theory did not attempt to dismiss the Universe's expansion [81] - [83]. Instead, it states that the Universe does not change its appearance over time despite that it is expanding [81] - [83].

In any case, it must be stressed that, physically, the Big-Bang, was not a conventional explosion, which is a different claim than that of Santilli and collaborators [19] - [22]. In order to clarify this and other misunderstandings, mistakes and flaws on the standard model of cosmology in [19] - [22], let us start to resume the FLRW solution following the lines of [10, 24]. In Keel's words [84] the cosmological principle states that "Viewed on a sufficiently large scale, the properties of the Universe are the same for all observers". The Universe appears statistically homogeneous on scales larger than 250 million light years, although it is inhomogeneous at smaller scales [85]. This is confirmed by the Cosmic Microwave Background (CMB), which is isotropic, i.e., the CMB intensity is about the same in all directions that we look at [85]. The space-time's geometry corresponding to a homogeneous and isotropic Universe is given by the FLRW line element (we use again geometrized units) [10, 24] 


$$
d s^{2}=a^{2}(\eta)\left[d \eta^{2}-d \chi^{2}-\Sigma(\chi)^{2}\left(d \theta^{2}+\sin ^{2} \theta d \varphi^{2}\right)\right],
$$

corresponding to three different possibilities [10,24]

$$
\begin{array}{ccc}
\chi & \\
\Sigma(\chi) \equiv \sin \chi & \\
\sinh \quad & \chi & (3)
\end{array}
$$

and the conformal time $\eta$ is called the time arch-parameter [24]. The derivation of the FLRW line element from the assumption of a homogeneous and isotropic Universe can be found in various textbooks - such as, for example [10]. Setting $\eta=$ constant we get the spatial sections as [24]

$$
d s^{2}=a^{2}(\eta)\left[d \chi^{2}+\Sigma(\chi)^{2}\left(d \theta^{2}+\sin ^{2} \theta d \varphi^{2}\right)\right] .
$$

Thus, although we have the spherical rotational symmetry in $\theta$ and $\varphi$, we only find a Euclidean geometry for case (1) of eq. (35). In fact, the length in the $\chi$ direction is given by [24]

$$
d l_{\text {rad }}=a d \chi,
$$

which implies that the length of the circumference having $\chi=$ constant is [24]

$$
l_{\text {circ }}=2 \pi a \Sigma(\chi)
$$

that is Euclidean only for case (1). One can also introduce the cosmic time as [24]

$$
t \equiv \int a(\eta) d \eta
$$

transforming eq. (34) into

$$
d s^{2}=d t^{2}-a^{2}(t)\left[d \chi^{2}+\Sigma(\chi)^{2}\left(d \theta^{2}+\sin ^{2} \theta d \varphi^{2}\right)\right] .
$$

Putting $\sigma \equiv \Sigma(\chi)$ in eq. (40) one gets [24]

$$
d s^{2}=d t^{2}-a^{2}(t)\left[\frac{d \sigma^{2}}{1-k \sigma^{2}}+\sigma^{2}\left(d \theta^{2}+\sin ^{2} \theta d \varphi^{2}\right)\right],
$$

with $k=0,-1,1$ for the cases (1), (2) and (3) in eq. (35), respectively [24].

On the other hand, setting $r \equiv 2 a \tan \left(\frac{\chi}{2}\right)$ and using the standard transformations from polar to cartesian coordinates one can rewrite eq. (40) to obtain the FLRW isotropic line element [24]

$$
d s^{2}=d t^{2}-a^{2}(t) \frac{d x^{2}+d y^{2}+d z^{2}}{1+k \frac{r^{2}}{4 a^{2}}},
$$


where we understand that the name "isotropic" is due to the expression $d x^{2}+$ $d y^{2}+d z^{2}$, which immediately shows the isotropy of the spatial metric around each point, while the scale factor $a$ depends on $r$ [24].

Now, we discuss the propagation of the light in the FLRW geometry. For the sake of simplicity, we will discuss only the "radial" propagation, i.e. we will set both $\theta$ and $\varphi$ as constants. It is well known that this is not a restriction [24]. From eq. (34) we get [24]

$$
d \eta= \pm d x \quad \Longrightarrow \quad \eta= \pm x+\text { const }
$$

Assuming that the light is emitted in $\left(\eta_{e}, x_{e}\right)$ and is received in $\left(\eta_{r}, x_{r}\right)$ - with $x_{r}>x_{e}$ - we obtain [24]

$$
\eta_{r}-\eta_{e}=x_{r}-x_{e}
$$

We stress that we are using comoving coordinates, which are coordinates assigning constant spatial coordinate values to observers who perceive the Universe as isotropic [24]. We recall that, along time-like geodesics the proper time is given by [24]

$$
d \tau=a(\eta) d \eta .
$$

Thus, considering two subsequent signals, we get [24]

$$
d \eta_{r}=d \eta_{e} \quad \Longrightarrow \quad \frac{d \tau_{r}}{d \tau_{e}}=\frac{a_{r}}{a_{e}} .
$$

Hence, we found a redshift's effect. Traditionally one sets $z \equiv \frac{\Delta \lambda}{\lambda}$ and, in turn, one gets [24]

$$
1+z=\frac{\lambda_{r}}{\lambda_{e}}=\frac{a_{r}}{a_{e}} \Longrightarrow z=\frac{a_{r}-a_{e}}{a_{e}} .
$$

This is called the cosmological redshift in order to distinguish it from the gravitational redshift and from the Doppler shift which is due to the particular motion of the sources [24]. Thus, we will have a real, concrete effect only if $a_{r}>a_{e}$. We will see below that this is guaranteed by the FLRW equations.

Let us consider the situation in which $x_{r}$ is not too much larger than $x_{e}$. In that case, $\eta_{r}$ is not too much larger than $\eta_{e}$ and we get [24]

$$
z \simeq \frac{1}{a} \frac{d a}{d \eta}\left(\eta_{r}-\eta_{e}\right)=\frac{d a}{d t}\left(x_{r}-x_{e}\right) .
$$

To first order, the distance $l$ between source and receiver is [24]

$$
l \simeq a\left(x_{r}-x_{e}\right)
$$

and we get

$$
z \simeq \frac{1}{a} \frac{d a}{d t} l=H l \text { with } H \equiv \frac{1}{a} \frac{d a}{d t} .
$$

Eq. (50) is the Hubble law and $H$ is the Hubble constant. The Hubble law states that the cosmological redshift is proportional to the distance from the radiation's source. We recall that, although this law is dedicated to the American 
astronomer Edwin Powell Hubble, who verified it through his astronomical observations [75], it was originally derived in a theoretical framework in 1927, i.e. two years before Hubble's paper, by Lemaître [76]. We also stress that the Hubble law is only a first order approximation for galaxies which are not too distant from each other. As today we know sources having $z>4$, better approximations are needed. For example, considering second order approximation, we find the parabolic relation [24]

$$
z=H_{0} l+K\left(H_{0} l\right)^{2},
$$

where $H_{0}$ is the present day's value of the Hubble constant and $K$ is a constant of proportionality - see [24] for details.

Now, we discuss the cosmological dynamics. For this, we require two additional pieces of information [24]:

1. a law of motion (the FLRW equations), which arises from the Einstein field equation of eq. (33);

2. an equation of state for the matter, which informs us of the density variation during the evolution of the Universe.

Let us derive the components of the Einstein tensor. From eq. (40) one computes the Christoffell symbols, the Riemann tensor and the Einstein tensor in a orthonormal basis representing the local Lorentz frame of the matter obtaining [24]

$$
\begin{gathered}
G_{t t}=3\left(\frac{\dot{a}}{a}\right)^{2}+\frac{3 k}{a^{2}} \\
G_{\chi \chi}=G_{\theta \theta}=G_{\varphi \varphi}=-2 \frac{\ddot{a}}{a}-\left(\frac{\dot{a}}{a}\right)^{2}-\frac{k}{a^{2}}
\end{gathered}
$$

where, again, $k=0,-1,1$ is the sign of the curvature of the spatial hypersurfaces and all the other components of the Einstein tensor are null [24]. In order to write down the stress-energy tensor we need some physical motivations which permit one to postulate the distribution of the matter. Assuming the Universe as statistically homogeneous and isotropic on the cosmological scale the matter consists of $[24,39,40,85]$

1. "dust" (galaxies and/or something else) having negligible interactions;

2. black body radiation (the $\mathrm{CBR}$ );

3. other massless and massive particles (gravitons and neutrinos).

Today we know that neutrinos have nonzero masses [86]. In fact, such as

1. the $\mathrm{CMB}$ radiation,

2. galaxy surveys, and

3. the Lyman-alpha forest 
all indicate that the summed masses of the three flavour states of neutrinos must be less than $0.3 \mathrm{eV}$ [87]. In any case, the distribution of matter is resumed by the model of a perfect fluid having the stress-energy tensor [24]

$$
\mathbf{T}=(\rho+p) \mathbf{u} \otimes \mathbf{u}-p g .
$$

Using the same orthonormal basis of the Einstein tensor the components of the stress-energy tensor are [24]

$$
\begin{gathered}
T_{t t}=\rho \\
T_{\chi \chi}=T_{\theta \theta}=T_{\varphi \varphi}=p .
\end{gathered}
$$

With the sole exception of the first instants after the Big-Bang, the exchanges of energy and impulse among the various kinds of matter are negligible [24]. As a consequence, we can separately apply the conservation law to the different kinds of matter [24]. Considering massive matter (barions and neutrinos) the the conservation of the number of particles implies the conservation of the rest mass. On the other hand, the volume is occupied by particles with a scale of $a^{3}$ [24]. Thus, one gets [24]

$$
\rho_{m} a^{3}=\text { constant }
$$

Concerning the radiation, one assumes that the energy associated with the number of photons and gravitons is conserved and takes the redshift into account [24]. Thus, one gets that the numerical density scales as $a^{-3}$ and the energy density needs of a further scaling as $a^{-1}$. Hence, one gets [24]

$$
\rho_{r} a^{4}=\text { constant. }
$$

Then, one writes down the total equation of state for the mass-energy as [24]

$$
\begin{gathered}
\rho_{t}(t)=\rho_{m}(t)+\rho_{r}(t)=\rho_{m 0}\left(\frac{a_{0}}{a(t)}\right)^{3}+\rho_{r 0}\left(\frac{a_{0}}{a(t)}\right)^{4} \\
p(t)=\frac{1}{3} \rho_{r 0}\left(\frac{a_{0}}{a(t)}\right)^{4} .
\end{gathered}
$$

Combining eq. (52) with eq. (54) we obtain the FLRW equations [24]

$$
\begin{gathered}
\left(\frac{\dot{a}}{a}\right)^{2}+\frac{k}{a^{2}}=\frac{8 \pi}{3} \rho \\
\frac{\ddot{a}}{a}+\frac{1}{2}\left(\frac{\dot{a}}{a}\right)^{2}+\frac{k}{2 a^{2}}=-4 \pi p .
\end{gathered}
$$

From eq. (58) we find an immediate consequence on $\rho$ and $p$ [24]. In fact, deriving the first equation of eq. (58) with respect to $t$ and eliminating $\ddot{a}$ through the second equation of eq. (58) one finds [24]

$$
\frac{d}{d t}\left(\rho a^{3}\right)+p \frac{d}{d t} a^{3}=0 .
$$


One can easily check that eq. (59) directly arises from the energy conservation $\nabla \cdot \mathbf{T}=0[24]$. On the other hand, this equation is contained in eqs. (55) and (56). In fact, one one hand $\rho_{m} a^{3}$ is constant for eq. (59) while $p_{m}=0$ [24]; on the other hand, eq. (56) together with $p_{r}=\frac{1}{3} \rho_{r}$ shows that both $p_{r}$ and $\rho_{r}$ satisfy eq. (59) [24]. Henceforth, one concludes that if we use eqs. (55) and (56), then only one of the FLRW equations is sufficient; although sometimes it may be beneficial to use both of them [24].

Now by recalling the definition of the Hubble constant in eq. (50) and using the first of the FLRW equations of eq. (58), then one gets [24]

$$
H^{2}=\left(\frac{\dot{a}}{a}\right)^{2}=\frac{8 \pi}{3} \rho-\frac{k}{a^{2}} \Rightarrow H_{0}^{2}=\frac{8 \pi}{3} \rho_{0}-\frac{k}{a_{0}^{2}},
$$

where the subscript 0 refers to the values at the present time, where the critical density is defined as the value of the density corresponding to $k=0$ (Euclidean spatial hyper-surfaces), i.e. [24]

$$
\rho_{c}=\frac{3}{8 \pi} H^{2} \Rightarrow \rho_{0 c}=\frac{3}{8 \pi} H_{0}^{2} .
$$

Recent measurements from the Planck mission indicate a value of $67(\mathrm{~km} / \mathrm{s}) / \mathrm{Mpc}$ for the Hubble constant [89], while observations are consistent with $k=0$ (flat Universe) to percent-level precision [90]. It is tradition to introduce the nondimensional parameter $\Omega$ as the ratio between the effective and the critical densities, i.e. [24]

$$
\Omega \equiv \frac{\rho_{t}}{\rho_{c}},
$$

which permits us to rewrite eq. (60) as [24]

$$
\frac{k}{a^{2}}=H^{2}(\Omega-1) \Rightarrow \frac{k}{a_{0}^{2}}=H_{0}^{2}\left(\Omega_{0}-1\right) .
$$

We note that for a flat Universe it is $\Omega=1 \Rightarrow \Omega_{0}=1$ [24].

Now, let us see the cosmological dynamics arising from the FLRW equations. For this, we need to assume particular choices for the equation of state for the matter [24]. We know that at the present time the pressure can be neglected, but this neglecting did not work when $a$ was small because the radiation's contribution scales as $a^{-4}$ and becomes dominant [24]. Then, it is reasonable to assume that the real evolution is included between the two extremal cases [24]

$$
\begin{gathered}
p=0 \quad \text { matter dominated Universe } \\
p=\frac{1}{3} \rho \quad \text { radiation dominated Universe. }
\end{gathered}
$$

We will discuss only the cases for which it is $k=0$, because they are in agreement with the most recent cosmological observations [90]. The other cases are discussed in detail in [24]. We will see that both of the models have a similar behavior. Thus, we expect that also the real character of the Universe's evolution should be similar. 
From eqs. (57) and (58), setting $k=0$ we get [24]

$$
\begin{gathered}
\left(\frac{\dot{a}}{a}\right)^{2}=\frac{8 \pi}{3} \rho \\
\rho a^{3}=\text { const. }=\rho_{0}\left(\frac{a_{0}}{a(t)}\right)^{3},
\end{gathered}
$$

having the solution $[24]$

$$
\begin{gathered}
a=\left(\frac{3 t}{2} \sqrt{a_{*}}\right)^{\frac{2}{3}} \\
a_{*} \equiv \text { integration constant },
\end{gathered}
$$

for the matter dominated Universe, and [24]

$$
\begin{gathered}
\left(\frac{\dot{a}}{a}\right)^{2}=\frac{8 \pi}{3} \rho \\
\rho a^{4}=\text { const. }=\rho\left(\frac{a_{0}}{a(t)}\right)^{4},
\end{gathered}
$$

having the solution $[24]$

$$
\begin{gathered}
a=\sqrt{2 a_{*} t} \\
a_{*} \equiv \text { integration constant },
\end{gathered}
$$

for the radiation dominated Universe, respectively. We note that in both of the models a Big-Bang singularity is present for $t=0$ [24]. In other words, there exists a past $t$ for which one gets $a=0$. Thus, here we clarify other basic misconceptions by Santilli and collaborators in [18]-[22]. First of all, we note that the Earth has not been cited in all our discussion, which was based on the cosmological principle while the FLRW equations have been developed through the Einstein field equations of eq. (33) in a generic orthonormal basis representing the local Lorentz frame of the matter [24]. Thus, claiming that "the expansion of the Universe implies a necessary return to the Middle Ages with Earth at the center of the Universe" [18]-[22] is a very basic, elementary mistake. Second, both of eqs. (66) and (68) imply an expanding Universe which arises from the application of the GTR, contrary to the claim that the GTR implies "the expected contraction due to gravitational attraction" [19, 22]. Third, in [19] Santilli and collaborators claim that "Conjecture 3: The universe was born in a primordial explosion, the "big bang", occurred some 13,7 billions years ago (see, e.g., Ref. [32]). This conjecture was voiced for the intent of maintaining the credibility of the expansion of the universe, thus implying that the claimed primordial explosion had to occur in the galactic vicinity of Earth and, consequently implying again that Earth is at the center of the universe" and that "As it is the case for all explosions without adaptations to preferred theories, the universe around Earth should be completely empty of any galaxy for up to 13.7 billion light years, and the "debris" of the claimed explosion (the galaxies) should 
slow down with the increase of the distance due to inevitable intergalactic dust. Both incontrovertible implications of any explosion, including the big bang, are dramatically disproved by astronomical evidence". In [22] Santilli adds that "By the very definition of "explosion" according to the Webster Dictionary, in the event the universe originated from a primordial explosion, our galactic environment should have no galaxies for 13.7 billions of light years or, in any case, the "debris" of the primordial explosion should have created a region of empty space around Earth. This implication is disproved by astrophysical evidence on the distribution of galaxies in our environment; Also according to the very definition of "explosion,"the speed of its "debris" (the galaxies) should decrease with the increase of the distance, as a result of which the big bang conjecture is incompatible with the very assumption of the expansion of the universe and its acceleration with the distance for which representation the conjecture was proposed". Clearly, these are other very basic, elementary misconceptions. In fact, we did not use conjectures, but, instead, we applied the GTR to the cosmological principle and to the cosmological observations. We have shown that the Big-Bang was not a primordial explosion, but it was instead a sort of "emerging" of the whole space-time from infinitesimal dimensions, according to the FLRW equations.

In order to finalize our discussion on the FLRW cosmology, we now discuss the problem of the singularity. In fact, in [22] Santilli also claims that "The big bang conjecture provides no consistent model for the origin of the universe since the infinities that are inherent in a conjectured geometric singularity prevent any quantitative study of the real origin of the universe, not to mention the lack of addressing of what type of universe existed prior to the explosion." First of all, we stress that we stress that time also arises from the Big-Bang singularity in FLRW cosmology [24]. On the other hand, it is well known that today some bouncing models are proposed that permit the removal of the singularity in order to go back in time before the precise instant of the Big-Bang - see for example [91]. In such models the Big-Bang is considered to be a time in the Universe's past history in which, although the density and the curvature were extremely high, they were not infinity [91]. For the sake of technical correctness, let us recall the rigorous definition of singularity by Schmidt [92]: "To any space time a boundary is attached on which incomplete geodesics terminate as well as inextensible timelike curves of finite length and bounded acceleration. The construction is free ofad hoc assumptions concerning the topology of the boundary and the identification of curves defining the same boundary point. Moreover it is a direct generalization of the Cauchy completion of positive definite Riemannian spaces". One could think that the existence of the singularity in the FLRW cosmologies could be a peculiarity of those models, due to the high symmetry arising from the cosmological principle [24]. As a consequence, one could suspect that even low displacements from the perfect symmetry could remove the initial Big-Bang singularity. Roughly speaking, if the geodesics of matter are no more than all radial (in the sense of the 4-dimensional space-time), one is not sure that all of them must converge in the singular termination point when one looks back in time [24]. This problem has been well addressed by the Hawking- 
Penrose singularity theorems, which have shown that, under "resonable physical assumptions", a singularity cannot be avoided from a physical point of view [24]. On the other hand, the presence of a quintessential density term in the model in [91], which is due to a particular non-linear electrodynamics Lagrangian used to construct the stress-energy tensor in the R. H. S. of the Einstein field equation of eq. (33), permits one to violate the "resonable physical assumptions" of the Hawking-Penrose theorems, generating a non-singular cosmology. We additionally stress that in general, the GTR is a classical theory, which does not take quantum effects into account [24]. It is well known that such quantum effects become very important at the Planck scale [24], i.e. for densities of order of the Planck density $10^{93} \frac{\mathrm{g}}{\mathrm{cm}^{3}}$. When we insert this condition in both the matter dominated and radiation dominated models we get a time on the order of Planck time, namely $10^{-43} s$ [24]. Thus, the GTR does not work for times which are near the supposed initial Big-Bang singularity. It is a general opinion that a solution should be a quantum theory of gravity, unifying the GTR with quantum mechanics (QM). This goal has not yet been achieved.

Santilli and collaborators have also released wrong claims on the Dark Energy issue, see for example [19], where the authors claim "Conjecture 6: The dynamics of the entire universe depends on yet an additional invisible substance, the "dark energy", constituting over 95\% of the energy in the universe (see e.g., Ref. [36]). This yet additional conjecture was voiced for the generally unspoken insufficiencies of the big bang conjecture (some of which are recalled below), to explain the expansion and the acceleration of the expansion of the universe, with ensuing need for enormous amounts of energy" and that "the conjecture of dark energy has been voiced in support of the preceding far reaching conjecture of the acceleration of the expansion of the universe, since the latter required an amount of energy simply beyond our imagination; the relationship between the conjecture of dark energy and the conjecture of the big bang has remained essentially unspoken, since the claim of the representation of the expansion of the universe via the big bang has remained untouched following the conjecture of dark energy; as it was the case for the dark matter, a uniformly distributed dark energy cannot provide any measurable effect on the dynamics of an individual galaxy". Here, we again find various basic, elementary misconceptions, so let us clarify this issue. The key point is that, although the FLRW cosmological model, which is based on the GTR, can explain the Universe's expansion (as we have shown above), it cannot explain the Universe's acceleration, which is today confirmed by all the cosmological observations [36]-[40]. In the standard cosmological model - the $\Lambda$ CDM "concordance model" - the FLRW field equations are extended by a new source which is inserted in their right side hand. This new ingredient is the so-called Dark Energy, which gives a large negative pressure [36]-[40]. From the geometric point of view, the Dark Energy is introduced by reinserting the cosmological constant [73] in the Einstein field equations of eq. (33). Thus, eq. (33) becomes the one that Aczel calls "God's Equation" [93]

$$
G_{i k}+\Lambda g_{i k}=8 \pi T_{i k} .
$$

In other words, the cosmological constant generates a space-time curvature 
which depends neither on the standard stress-energy tensor of the matter nor on the gravitational waves. Although many things about the nature of the Dark Energy remain a matter of speculation, we stress that, contrary to the claims by Santilli and collaborators [19], in order to explain the acceleration of the expansion of the Universe one does not require an enormous amount of energy. In fact, in the framework of the the $\Lambda \mathrm{CDM}$ model the Universe is made by $26.8 \%$ Dark Matter, $68.3 \%$ Dark Energy (for a total of $95.1 \%$ ) and $4.9 \%$ ordinary matter [89]. On a mass-energy equivalence basis, the density of Dark Energy is today $6.91 \times 10^{-27} \frac{\mathrm{g}}{\mathrm{cm}^{3}}[89]$. This is a very low value, much less than the density of ordinary matter or Dark Matter within galaxies. Surely, it is not "an amount of energy simply beyond our imagination" as erroneously claimed by Santilli and collaborators [19]. In other words, the effect of the Dark Energy is merely a small constant negative pressure of vacuum. The reason because Dark Energy dominates the mass-energy of the Universe is that it is uniform across space [94]. Notice that in [21] it is claimed that "Dark Energy cannot possibly provide any measurable effect when equally distributed". This is another elementary mistake. In fact, the Dark Energy operates directly on the evolution of the scale factor of the Universe $a$ [94]. Clearly, if such an evolution is accelerated, the whole Universe is accelerating. In [22] Santilli claims that "no model expected to pass the test of time has been provided to the author's best knowledge on how energy could cause gravitational repulsion". Instead, it is very simple to provide such a model. The key point is that the gravitational interaction between masses - which remains attractive - is not influenced by the negative pressure due to the Dark Energy. That negative pressure indeed alters the overall evolution of the Universe at the cosmological scale as it acts directly on the scale factor $a$ and, in turn, results in the accelerating expansion of the Universe despite the attraction among the masses present in the Universe.

Another point to be clarified concerns the claims by Santilli and collaborators [19] that "We should also mention the implausibility of the expansion of the universe because the speed needed for the representation of the cosmological redshift of galaxies at the edge of the known universe is today approaching and then surpassing the speed of light in vacuum, namely, the conjecture here considered implies that billions of galaxies travel at superluminal speeds!". Let us clarify this point. Assuming that the acceleration will continue indefinitely, one finds that galaxies outside the local supercluster will have line-of-sight velocities continually increasing with time. Eventually, those velocities will exceed the speed of light [95]. The key point is that this is not a violation of the STR. In fact, the notion of "velocity" used here is different from the one in a local Lorentz frame, which must be constrained to be less than the speed of light for any massive object. As the Hubble constant decreases with time, we can find cases where galaxies receding from us faster than light can emit a signal which can, in principle, reach the Earth [96]. On the other hand, based on the accelerated expansion, there are galaxies which could cross a sort of cosmological event horizon. In that case, any emitted light by such galaxies which will past that point, will be unable to reach the Earth at any time in the future [97]. In fact, the light cannot reach a point where its "peculiar velocity" toward us is 
higher than the expansion velocity away from us [97]. The current distance to this cosmological event horizon should be about 16 billion light years [96]. In other words, a signal from an event happening at the present time can be able to reach the Earth in the future only if the event were less than 16 billion light years away. Instead, the signal cannot reach the Earth if the event were more than 16 billion light years away [96].

When a galaxy approaches the point of crossing of the cosmological event horizon, the light from it will become more and more redshifted, arriving to a point where the frequency becomes too small to be detected. As a consequence, the galaxy appears to vanish completely [95]. In any case, the Earth, the Milky Way, and galaxies which are not too far will remain visible, while the rest of the Universe will recede and continue to disappear from view.

Another wrong claim by Santilli and collaborators concerns the CBR [19]: "the conjecture that the background radiation is "evidence" of the big bang has been disproved by calculations showing that, due to its weakness, such a radiation should have been absorbed by intergalactic media and galaxies billions of years ago". We note that the authors of [19] do not show such calculations, which indeed are surely wrong. In fact, the CBR temperature is well known to be very low, on the order of $2.7 \mathrm{~K}$ [98]. When gases fall into the intergalactic medium from the voids - which are the huge spaces between galaxy clusters - they heat up to temperatures of $10^{5} \mathrm{~K}$ to $10^{7} \mathrm{~K}$ [99]. On the other hand, ordinary stars have surface temperatures in the range from $10^{3} K$ to $10^{4} K$ [100]. Thus, in order to "have been absorbed by intergalactic media and galaxies" the CBR must violate the second law of thermodynamics!

Finally, we discuss the claims by Santilli and collaborators that "As well known since the time of Hubble's discovery, intergalactic space is far from being empty, since it is constituted by a medium with high energy density characterized by light (originating from all of the universe), gases, particles, radiations, etc. As established experimentally on Earth [8-10], such a medium causes a mutation of spacetime into the Minkowski-Santilli isospacetime, with consequential inapplicability (at said intergalactic distances) of special relativity in favor of the covering isorelativity (ITR) and resulting iso-redshift (IRS) without a necessary relative motion between the source, the medium and the observer (see below for the case with $v=0$ ). It is evident that the IRS eliminates any need for the expansion of the universe since light merely loses energy $E=h \nu$ to the very cold intergalactic medium, resulting in a numerical and time invariant (thanks to the LPS isosymmetry), representation of the cosmological redshift of galaxies without any necessary motion away from us" [19]. Here the key point is that Santilli and collaborators [18] - [22] assume that in a non-vacuum medium there should be deviations from the STR, which they suppose to be strictly valid only in vacuum, in favour of the so-called IRT of Santilli. One of the consequences of the IRT is that "shifts of the frequency of light propagating within a gaseous medium without any necessary relative motion between the source, the medium and the observer" [19] should exist. It is not our intention here to make claims on the correctness or on the lack of correctness of the IRT and of the consequent IRS. We will merely show that the effect of the IRS is $10^{-6}$ 
smaller than the effect requested to achieve the cosmological redshift. Let us start by recalling that Santilli and collaborators claim that the redness of the sun at sunset and sunrise is due to the IRS instead of the classical Rayleigh scattering mechanism [18] - [22]. This hypothesis is very speculative, to say the least, and has been ultimately shown to be wrong by Gandzha in [101]. In any case, Santilli and collaborators [18] - [22] claim that, as the redness of the sun at sunset and sunrise is due to the IRS, this should imply that the IRS can achieve the cosmological redshift because "intergalactic space is far from being empty, since it is constituted by a medium with high energy density characterized by light (originating from all of the universe), gases, particles, radiations, etc." [19]. Even assuming that Santilli and collaborators are correct in claiming that the redness of the sun at sunset and sunrise is due to the IRS, this is a completely non-scientific way of reasoning. In fact, in [18] - [22] Santilli and collaborators did not quantitatively support their claims but released a merely qualitative discussion. This has to do neither with the scientific method, nor with a scientific way to obtain dynamical equations and causal structures from observations. Let us neglect the correct analysis by Gandzha in [101] and, instead, let us assume that the data on the IRS in [19] are correct. Thus, the redshift detected in [19] at sunset is about $z=0.2$ for an approximate $200 \mathrm{~km}$ passage through the atmosphere. The density of air is about $10^{-3} \frac{\mathrm{g}}{\mathrm{cm}^{3}}$. We will assume a density's value half of this one. In fact, we need to take account that much of the solar beam passes through more rarified air. So the sunlight would be passing through a column density of $\sim 10.000 \frac{\mathrm{g}}{\mathrm{cm}^{2}}$. One estimate of the intergalactic hydrogen density given by Crawford [102] is 1.4 hydrogen atoms per $\mathrm{m}^{3}$. This is equivalent to $2 * 10^{-30} \frac{\mathrm{g}}{\mathrm{cm}^{3}}$. A distance of $1 \mathrm{Mpc}$ measures to be $5.5 * 10^{23} \mathrm{~cm}$ and so there would be a column density of about $10^{-6} \frac{\mathrm{g}}{\mathrm{cm}^{2}}$ per megaparsec. This is 10 billion times smaller than the redshift that the authors of [19] measured in the Earth's atmosphere. So the contribution of this IRS effect to the cosmological redshift would be $z \sim 2 * 10^{-10} / M p c$. By comparison the Hubble constant is around $z=2.4 * 10^{-4} / M p c$. So the IRS would be $10^{6}$ fold smaller and hence can be completely neglected. We add that gas column densities cited for clouds enveloping some quasars range up to $5 * 10^{21}$ hydrogen atoms per $\mathrm{cm}^{2}$ [103]. So for hydrogen, this amounts to a column density of $\sim 8 * 10^{-3} \frac{\mathrm{g}}{\mathrm{cm}^{2}}$. This is $\sim 8 * 10^{-7}$ smaller than the column density in the Sunset experimental data in [19]. So it would contribute a redshift of up to about $z=2 * 10^{-7}$, or $\sim 0.05 \mathrm{~km} / \mathrm{s}$, again completely negligible. Thus, through this relatively easy argument, we've ultimately shown that: even assuming that the redness of the sun at sunset and sunrise is due to the IRS, this cannot explain the cosmological redshift - proving that the claims of Santilli and collaborators in [18] - [22] are wrong. It immediately follows that the claims of Santilli and collaborators in [18] - [22] - which dismiss the expansion of the Universe - are completely wrong and unscientific. Clearly, if such a weak IRS signal cannot dismiss the expansion of the Universe, it also dismisses neither the accelerated expansion nor the presence of the Dark Energy, for which a stronger signal is needed. This is again in opposition to the very wrong claims in [18] - [22]. 


\section{Conclusion and Outlook}

Finally, to conclude this work and results, we briefly recall some paramount issues in the physics of gravitation and cosmology, and recapitulate how this unscientific, unprofessional, undisciplined confusion generated by the Santilli club is being reconciled with the methods and practices of science. In our opinion, given these circumstances, this outlook may serve as a meaningful reminder.

At the heart of physics and the mathematical sciences is this long-lived problem of grand unification: to rigorously characterize the laws of nature and consolidate the four fundamental interactions of the Universe into a single, unified field theory that can be experimentally-verified in the laboratory and thereafter applied to possibly limitless applications. Solving this gigantic, convoluted problem and constructing such a framework of gravitation and cosmology are decisive prerequisites for the general advancement and furthered application of chemistry, biology, medicine, mathematics, computing, engineering, and many other disciplines that admit the far-reaching potential to beneficially transform the planet and reveal the cosmological origin of the Universe. Indeed, it is not difficult to argue that this fundamental problem stands as one of the mightiest challenges in the recorded history of the totality of science; it has undeniably vexed and challenged the minds of observers and scientists for countless generations.

In the said disciplines, it is well known that a powerful, indispensable toolbox for achieving groundbreaking discoveries and improvements is in fact the scientific method. One may define the scientific method as a set of strategies and practices for the investigation of phenomena, the acquisition of new knowledge, and/or the correction, integration, and application of previous knowledge. Once the questions are asked, then hypotheses must be proposed, examined, developed, refined, and ultimately tested via experiments that can be reproduced in similar conditions. One may think of the methods and practice of science - both theoretical and experimental - as being characterized by a collection of ongoing, dynamic, rigorous, step-by-step processes that interactively operate on a global scale to produce useful knowledge and results. Thus, in pursuit of a unified field theory equipped with an essential foundation of gravity and cosmology, researchers around the globe must continue to adhere to the key principles of the scientific method in order to contribute and propagate consistent, reliable, professional ideas, procedures, results, and applications to the scientific community.

In this work, we've applied the scientific method to thoroughly and systematically review, assess, and address the obscure claims and conjectures initiated by Santilli and collaborators in this realm of gravitational and cosmological unification; which specifically include numerous unscientific, strong statements against the GTR and the standard $\Lambda \mathrm{CDM}$ model, yet favor the so-called IGT and a non-expanding Universe. We've shown that the results are clear: such unscientific assertions and "results" are due to fundamental misunderstandings of very basic concepts of gravitation and cosmology, in which no vetted mathe- 
matical foundation exists and a profusion of experimental evidence is violated. In this case it is evident that, in general, the Santilli club needs to be reminded of the procedures and practices of science and professional research. Only a true application of the scientific method permits to obtain correct dynamical equations and causal structures from observations. Surely, this is not the case of Santilli and collaborators in [1] - [6], [11, 12], [18] - [22], [46, 58, 59, 62].

Now it is undoubtedly true that the Universe exhibits a plethora of mysterious phenomena for which many unanswered questions still exist. Moreover, it is also the case that frameworks such as the GTR and quantum mechanics do not independently qualify as unification candidates, and therefore require an additional "upgrade" with a rigorous mathematical treatment via the scientific method in order to reconcile the four fundamental interactions; indeed, we need not remind the reader that this issue has been the subject of major scientific research for many generations. For such classical, modern, and developing theories it is imperative to repeatedly question their capabilities, identify possible shortcomings, and propose corrections and alternative theories for experimental submission. In the procedures and practice of scientific professionals, no such clues, evidence, or data may be overlooked. Consequently, while some of Santilli's claims and work in other contexts may be scientific in nature, those that we've identified in this paper are surely not.

On one hand, Santilli has initiated some striking discoveries in other areas of science such as the new "MagneGas Fuel" [104] - [108] and "Intermediate Controlled Nuclear Synthesis (Fusion) [109] - [111]. Both of these relatively safe, clean, cost-effective alternative energy systems have been officially patented $[108,109]$ and are based on Santilli's theory of "Hadronic Mechanics"' (HM) [105]. To some extent, this HM theory has been experimentally-verified [107, $110,111]$ and, more recently, the resulting magnecule-based technologies have been elevated to the status of real-world industrial application and production such technologies are definitely worthy of additional investigation. We note that such technology could potentially transform and improve the energy sector and the economy on a global scale. But, on the other hand, the arcane, unscientific, unprofessional, undisciplined chaos generated by the Santilli club is not helping this case and convolutes the distinction between the IGT (without a scientific basis) and the HM (with an apparent scientific basis). Indeed, the problems associated with the mysteries of the Universe are so vast that no single scientist can be correct all of the time - mistakes, miscalculations, and misconceptions are sure to be made, and therefore such work should continue to be subjected to additional research via the scientific method.

Thus, in the case of gravitation and cosmology, we've shown that Santilli and cohorts have clearly discarded the scientific method, ignored the abundance of experimental evidence, and profusely created turmoil that is causing unnecessary problems. If the forces of the mathematical science community are to achieve a grand unification, then surely we must work together as a single united force and utilize the tools and methods of science to attack and conquer these great problems and mysteries of the Universe. 


\section{Acknowledgements}

Christian Corda thanks Mr. Ruggero M. Santilli and collaborators for asking him to write a paper criticizing Santilli's studies in gravitation and cosmology.

\section{Author Contributions}

The authors declare that each of them contributed in equal way to realize this paper.

\section{$7 \quad$ Conflict of Interests}

The authors declare that there is no conflict of interests regarding the publication of this article.

\section{References}

[1] R. M. Santilli, arXiv:physics/0611253v1.

[2] R. M. Santilli, "Isotopic Generalizations of Galilei's and Einstein's Relativities". Vol. I "Mathematical Foundations" and Vol. II "Classical Formulations", Hadronic Press Inc., Palm Harbor (1991).

[3] R. M. Santilli, Found. Phys. Lett. 10, 307 (1997).

[4] R. M. Santilli, arXiv:physics/9705016v1.

[5] R. M. Santilli, "Isodual Theory of Antimatter", Fundamental Theories of Physics 151, Springer, Dordrecht (2006).

[6] R. M. Santilli, Gal. Electr. 17, 4 (2006).

[7] C. M. Will, Living Rev. Relativity, 9, 3 (2006).

[8] P. Freud, Ann. Math. 40, 417 (1939).

[9] E. A. Notte-Cuello, W. A. Rodrigues Jr, Adv. Appl. Cliff. Alg. 19, 1, 113 (2009).

[10] L. Landau and E. Lifsits, Classical Theory of Fields (3rd ed.), London: Pergamon (1971).

[11] R. M. Santilli, http://www.i-b-r.org/scientific-rebirth-7.htm

[12] J. V. Kadeisvili, http://www.santilli-foundation.org/inconsistenciesgravitation.php

[13] S. Weinberg, "Gravitation and cosmology: principles and applications of the general theory of relativity", Wiley (1972). 
[14] S. Baessler, et al. Classical Quantum Gravity 18 (13) 2393 (2001).

[15] S. Baessler, et al., Phys. Rev. Lett. 83, 3585 (1999).

[16] R. von Eötvös, Math. Naturwiss. Ber. Ung. , 8, 65 (1890, in German).

[17] L. Iorio, Universe 1, 38 (2015).

[18] R. M. Santilli, AIP Proc. 1281, 882 (2010).

[19] R. M. Santilli, G. West and G. Amato, Journ. Comp. Met. Scien. Eng. 12,165 (2012).

[20] H. Ahmar, G. Amato, J. V. Kadeisvili, J. Manuel, G. West, and O. Zogorodnia, Journ. Comp. Met. Scien. Eng. 13, 321 (2013).

[21] R. Anderson, http://www.santilli-foundation.org/no-universeexpansion.php

[22] R. M. Santilli, Am. Journ. Mod. Phys. 4(2), 26 (2015).

[23] P. Gibbs, http://math.ucr.edu/home/baez/physics/Relativity/GR/centre.html

[24] C. W. Misner, K. S. Thorne and J. A. Wheeler, Gravitation (W. H. Feeman and Co., 1973).

[25] F. Zwicky, Proc. Nat. Ac. Sci. 15 (10), 773 (1929).

[26] G. Nordström, Phys. Zeit. 13, 1126 (1912)

[27] G. Nordström, Ann. Phys. Lpz. 42533 (1913).

[28] G. Nordström, Ann. d. Phys. 42, 533 (1913).

[29] C. Corda, New Adv. Phys. 7, 85 (2013).

[30] A. Einstein, Preuss. Akad. Wiss. Berlin, Sitzber., 778 (1915).

[31] C. Corda, Int. Journ. Mod. Phys. D, 18, 14, 2275 (2009).

[32] S. Nojiri and S.D. Odintsov, Int. J. Geom. Meth. Mod. Phys. 4, 115-146 (2007)

[33] S. Nojiri and S.D. Odintsov, Phys. Rep. 505, 59 (2011).

[34] A. Starobinsky, Phys. Lett. B, 91, 99-102 (1980).

[35] A. Pais, Subtle is the Lord, Oxford University Press (2005).

[36] A. G. Riess et al., Astron. Journ. 116, 1009 (1998).

[37] S. Perlmutter et al., Astrop. Journ. 517, 565 (1999).

[38] S. Cole et al., MNRAS 362, 505 (2005). 
[39] D. N. Spergel et al., Astrop. Journ. Supp. 170, 377 (2007).

[40] P. J. E. Peebles and B. Ratra, Rev. Mod. Phys. 75, 8559 (2003).

[41] C. M. Will, Theory and Experiments in Gravitational Physics, Cambridge Univ. Press Cambridge (1993).

[42] C. Brans and R. H. Dicke - Phys. Rev. 124, 925 (1961)

[43] M. Froeschlé, F. Mignard, and F. Arenou, in Proc. of Hipparcos Venice '97 Symposium (ESA, Noordwijk, Netherlands, 1997).

[44] P. G. Roll, R. Krotkov, R. H. Dicke, Ann. Phys., 26, 442 (1964).

[45] B. V. Braginski and V. I. Panov, Journ. Exp. Theor. Phys. 61, 873 (1971).

[46] R. Santilli, talk at the 12th International Conference of Numerical Analysis and Applied Mathematics, September 20154, Rodos Palace Hotel, Rhodes, Greece.

[47] R. Cox, Hadr. Journ. 35, 389 (2012).

[48] K. Schwarzschild, Sitzungsber. Preuss. Akad. Wiss. Berlin (Math. Phys.) 189 (1916).

[49] C. T Ridgely, Eur. J. Phys. 31, 949 (2010).

[50] C. Corda, EJTP 8, 25, 65 (2011).

[51] G. 't Hooft, http://www.staff.science.uu.nl/ hooft101/gravitating_misconceptions.html

[52] K. Schwarzschild, Sitz. Preu. Akad. Wiss. Berlin (Math. Phys.) 189 (1916).

[53] H. Reissner, Annal. Physik 50, 106 (1916).

[54] G. Nordström, Ver. Ko . Ned. Akad. Wet., Afdel. Natuurk. 26, 1201 (Amsterdam 1918).

[55] H. Bondi, F.A.E. Pirani, and I. Robinson, Proc. R. Soc. London A 251, 519 (1959).

[56] J. R. Oppenheimer and G. B. Volkov, Phys. Rev. 55 (4), 374 (1939).

[57] P. Boonserm, M. Visser, and S. Weinfurtner, Phys. Rev. D 71, 124037 (2005).

[58] Private communications with R. M. Santilli

[59] R. M. Santilli, http://www.santilli-foundation.org/docs/isogravitation.pdf

[60] K. Schwarzschild, Sitz. Preu. Akad. Wiss. Berlin (Math. Phys.) 424 (1916).

[61] C. Corda, Ann. Phys. 355, 360 (2015). 
[62] R. M. Santilli, comment at the 12th International Conference of Numerical Analysis and Applied Mathematics, 22-28 September 2014.

[63] A. L. Kholmetskii, T. Yarmanb, M. Arikc and O.V. Missevitchd, AIP Conf. Proc. 1648, 510011 (2015).

[64] W. Kündig, Phys. Rev. 129, 2371 (1963).

[65] A. L. Kholmetskii, T. Yarman and O. V. Missevitch, Phys. Scr. 77, 035302 (2008).

[66] A. L. Kholmetskii, T. Yarman, O.V. Missevitch and B. I. Rogozev, Phys. Scr. 79, 065007 (2009).

[67] R. L. Mössbauer, Zeitschrift für Physik A (in German) 151, 124 (1958).

[68] The BICEP2 Collaboration, Phys. Rev. Lett. 112, 241101 (2014).

[69] The Planck Collaboration, Astron. Astrop. (2015 ), http://dx.doi.org/10.1051/0004-6361/201425034

[70] C. Corda, Gen. Rel. Grav. 42, 1323 (2010); Erratum-ibid. 42, 1335 (2010).

[71] R. A. Hulse, J. H. Taylor, Astrop. J. Lett. 195, L51 (1975).

[72] A. Giazotto, Journ. Phys. Conf. Series, 120, 032002 (2008).

[73] A. Einstein, Sitzun. Pre. Akad. Wiss. Berlin (Math. Phys.) 142 (1917).

[74] A. Friedmann, Zeit. Phys.21, 326 (1924).

[75] E. Hubble, Proc. Nat. Acad. Sci. USA 15, 168 (1929).

[76] G. Lemaître, Ann. Soc. Sci. de Bruxelles, 47, 49 (1927), English translation in G. Lemaître, MNRAS, 91, 483 (1931).

[77] H. P. Robertson, Phil. Mag. Series 7, 5, 31, 835 (1928).

[78] A. G. Walker, Proc. London Math. Soc. s2-42 (1), 90 (1937).

[79] G. Gamow, "My World Line : An Informal Autobiography", Hardcover (1970).

[80] S. Mitton, "Fred Hoyle a life in science", Cambridge University Press (2011).

[81] F. Hoyle, MNRAS 108, 372 (1948).

[82] T. Gold and H. Bondi, MNRAS 108, 252 (1948).

[83] F. Hoyle and J.V. Narlikar, MNRAS 123, 133 (1961).

[84] W. C. Keel, "The Road to Galaxy Formation" (2nd ed.), Springer-Praxis (2007). 
[85] M. I. Scrimgeour et al., MNRAS 425, 116 (2012).

[86] G. Karagiorgi, A. Aguilar-Arevalo, J. M. Conrad, M. H. Shaevitz, K. Whisnant, M. Sorel, and V. Barger Phys. Rev. D 75, 013011 (2007).

[87] A. Goobar, S. Hannestad, E. Mörtsell and H. Tu, JCAP 06, 019 (2006).

[88] A. Macdonald, "General Relativity in a Nutshell", Luther College (2012).

[89] The Planck Collaboration, A\&A, 571, A1 (2014).

[90] The Planck Collaboration, A\&A, 571, A16 (2014).

[91] C. Corda and H. Mosquera Cuesta, Astropart. Phys. 34, 587 (2011).

[92] B. G. Schmidt, Gen. Rel. Grav. 1, 269 (1971).

[93] A. D. Aczel, "God's Equation: Einstein, Relativity, and the Expanding Universe", Four Walls Eight Windows (1999).

[94] B. W. Carroll and D. A. Ostlie, "An Introduction to Modern Astrophysics", 2nd Ed., Pearson Eduction, Inc. (2007).

[95] L. M. Krauss and R. J. Scherrer, Gen. Rel. Grav. 39, 1545 (2007).

[96] C. H. Lineweaver and T. M. Davis, Scient. Am. 292, 3, 36 (2005).

[97] A. Loeb, Phys. Rev. D 65, 047301 (2002).

[98] D. J. Fixsen, Astrophys. J. 707, 916 (2009).

[99] A. Gupta, M. Galeazzi, E. Ursino, Bull. Am. Astron. Soc. 41, 908 (2010).

[100] M. A. Zeilik and S. A. Gregory, "Introductory Astronomy $\& 3$ Astrophysics" (4th ed.). Saunders College Publishing (1998).

[101] I. Gandzha, Hadr. Journ. 35, 4, 379 (2012).

[102] D. F. Crawford, Aust. J. Phys., 40, 459 (1987)

[103] J. A. Rogerson, P.B. Hall, S.A. Snedden, M.S. Brotherton, S.F. Anderson, New Astr. , 16, 128 (2011).

[104] http://magnegas.com/

[105] R. M. Santilli, "Foundations of Hadronic Chemistry: With Applications to New Clean Energies and Fuels", Springer Science \& Business Media 120 (2001).

[106] C. P. Pandhurnekar, AIP Conf. Proc. 1648, 510015 (2015).

[107] Y. Yang, J. V. Kadeisvili and S. Marton, Int. Journ. Hydr. En. 38, 12, 5003 (2013). 
[108] R. M. Santilli, "Method and Apparatus for the Industrial Production of New Hydrogen-Rich Fuels", US Patent App. 14/244,229, Google Patents (2014).

[109] R. M. Santilli, "Method and apparatus for intermediate controlled fusion processes", US Patent App. 13/197,836, Google Patents (2011).

[110] J. V. Kadeisvili, C. Lynch and Y. Yang, Op. Phys. Chem. Journ. 5, 17 (2013).

[111] R. M. Santilli, "Experimental confirmation of the novel 'Intermediate Controlled Nuclear Fusion' without harmful radiations", New Adv. Phys. (2010). 